\title{
Effect of tellurium concentration on the structural and vibrational properties of phase-change Ge-Sb-Te liquids
}

\author{
H. Flores-Ruiz, ${ }^{1}$ M. Micoulaut, ${ }^{1}$ M.-V. Coulet, ${ }^{2}$ A. A. Piarristeguy, ${ }^{3}$ M. R. Johnson, ${ }^{4}$ G. J. Cuello, ${ }^{4}$ and A. Pradel ${ }^{3}$ \\ ${ }^{1}$ Sorbonne Universités, UPMC Université Paris 06, UMR 7600, LPTMC, F-75005 Paris, France \\ ${ }^{2}$ Aix-Marseille Université, CNRS, MADIREL UMR 7246, Campus de Saint jérôme, 13397 Marseille Cedex 20, France \\ ${ }^{3}$ Institut Charles Gerhardt, UMR 5253-CNRS, Case 1503, Université de Montpellier, Place Eugène Bataillon, \\ 34095 Montpellier Cedex 5, France \\ ${ }^{4}$ Institut Laue Langevin, BP 156, 6 rue Jules Horowitz, 38042 Grenoble Cedex, France
}

(Received 17 July 2015; published 12 October 2015)

\begin{abstract}
The structural properties of several compositions of $\mathrm{Ge}-\mathrm{Sb}$-Te liquids are studied from a combination of neutron diffraction and density functional-based molecular dynamics. We investigate structural properties including structure factors, pair distribution functions, angular distributions, coordination numbers, neighbor distributions, and compare our results with experimental findings. Most noticeable is the excellent agreement found in the reproduction of the structure in real and reciprocal spaces, resulting from the incorporation of dispersion forces in the simulation. The vibrational density of states is also measured from inelastic neutron scattering for different compositions and compared to the simulated counterpart, which exhibits an excellent agreement at low frequency.
\end{abstract}

DOI: 10.1103/PhysRevB.92.134205

PACS number(s): 61.25.Em, 61.20.Ja, 71.15.Pd

\section{INTRODUCTION}

Disordered tellurides have been the source of important applications and intense research in the last decade. They are considered as promising materials for phase-change memory (PCM) devices [1] and infrared transmitting wave guides [2]. For both applications, the disordered phases (liquid and amorphous) play a crucial role since they are involved either in the fabrication process or in the operation of the devices. For example, in the case of phase change materials, the data storage is based on the differences in structural and electronic properties between crystalline and amorphous phases. The process generally admitted is the following one: the initial crystalline material is rapidly heated above its melting point using an intense heating pulse (electrical or optical depending on the chosen application). The short and intense duration of the pulse quenches the material into its amorphous state at the submicrometer scale. It can then be reverted into its crystalline state using a less intense but longer pulse that heats the material just above its glass transition. This illustrates the fundamental role of the disordered phases: the amorphous state is important for the storage capability of the process; the liquid state is linked to amorphization and recrystallization processes that are crucial for the functionality of the devices. Amorphization is achieved by quenching the liquid, while the fast recrystallization of amorphous regions takes place above the glass transition, in the supercooled liquid state.

While the crystalline and amorphous phases have been extensively studied [1], much less work has been done on the structural and vibrational properties of such phase change liquids. The few studies published on liquid structure and dynamics have nevertheless confirmed the importance of the melt properties for the understanding of the amorphous state. From neutron diffraction experiments [3], the liquid structure was shown to be a good starting point to describe the amorphous phase, and it was shown that the liquid phase structure provides useful information on the suitability of a chalcogenide to be a good phase-change material. All materials with proven phase-change capabilities possess an octahedral local order and the densities of the amorphous and liquid phases were shown to be slightly lower than that of the crystalline phase. This result was later on confirmed for the $\mathrm{Ge}_{2} \mathrm{Sb}_{2} \mathrm{Te}_{5}$ (GST225) composition using X-ray absorption spectroscopy [4] (XAS). Based on extended x-ray absorption fine structure (EXAFS, geometric structure) and X-ray absorption near-edge structure (XANES, electronic structure) studies, the authors demonstrated that the local structure of amorphous and liquid phases were very similar. Moreover, the proposed covalent nature of the bonds between the elements was argued to explain the semiconducting behavior of the liquid. The vibrational properties were studied in the liquid state for two typical phase change materials $\mathrm{Ge}_{2} \mathrm{Sb}_{2} \mathrm{Te}_{5}$ and $\mathrm{Ge}_{1} \mathrm{Sb}_{2} \mathrm{Te}_{4}$ [5]. It was shown that, as for the structure factors [6], the vibrational densities of state are not temperature dependent in the liquid state, and this was interpreted as a consequence of the normal evolution of density in the liquid state, in contrast with Te-rich Ge-Te liquids.

From a theoretical viewpoint, there has been a vast body of studies using $a b$ initio simulations of the liquid state using density functional theory (DFT). Target compositions using the three elements have been mostly selected [5-11] on the compositional join having the best properties regarding phase change materials, i.e., on a pseudoternary line in the Ge-Sb-Te triangle defined by $\mathrm{GeTe}-\mathrm{Sb}_{2} \mathrm{Te}_{3}$ (Fig. 1). Apart from this tie-line, we are only aware of a numerical study of liquid $\mathrm{Ge}_{8} \mathrm{Sb}_{2} \mathrm{Te}_{11}$ [12] and $\mathrm{Ge}_{4} \mathrm{Sb}_{1} \mathrm{Te}_{5}$ [13]. Regarding the structure of these $\mathrm{Ge}_{2} \mathrm{Sb}_{2} \mathrm{Te}_{5}$ liquids, besides the outcome leading to an insightful full atomic scale picture, one of the remaining drawbacks of such methods is an underestimation of the intensity of the second and third main peak of the total structure factor $S(k)$, as well as a shift to lower wave vectors $[6,8]$. In addition, in real space, a systematic overestimation from DFT of the bond distances (and especially Ge-Te) has led to an important mismatch between the measured and the calculated total pair correlation function $g(r)$. This furthermore leads to coordination numbers that seem to be overestimated, at least when compared to alternative modeling schemes [16] or, more 


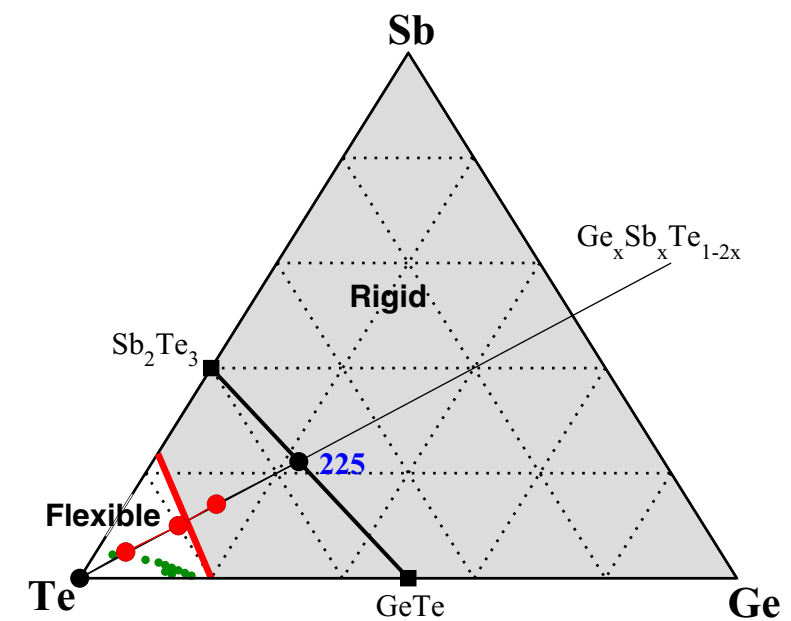

FIG. 1. (Color online) Compositional Ge-Sb-Te triangle. Red circles represent the compositions that are studied in this contribution by a combination of MD and neutron diffraction, black circles (Te and 225) are only studied by molecular simulations. Green symbols represent bulk glasses obtained from melt quench [14], while the red line represents the predicted rigidity transition line [15], which separates the triangle into a flexible phase and a stressed rigid phase (gray).

recently, to spectroscopic data [17]. Finally, the local geometry of Ge atoms, tetrahedral or defect octahedral, seems to be strongly affected by such bond distance overestimations, as recently demonstrated for a parent telluride glassy system [18]. Alternative techniques have been proposed to refine the structure of the amorphous phases such as Reverse Monte Carlo [19] or isochemical atomic substitution [20,21] but, to our knowledge, they were not yet used in Ge-Sb-Te melts.

The aim of this contribution is a deeper understanding of both structural and vibrational properties in Ge-Sb-Te liquids with high Te content. Our choice to concentrate on the Te-rich part of the phase diagram was motivated by recent results identifying possible flexible to rigid transition in the $\mathrm{Ge}-\mathrm{Sb}-\mathrm{Te}$ system. It was, indeed, shown that the phase diagram of this ternary alloy can be divided into two regions having different mechanical properties at the atomic scale: an underconstrained (flexible) phase for high Te content (see Fig. 1) and an overconstrained (stressed rigid) one [15] when the density of $(\mathrm{Ge}, \mathrm{Sb})$ cross-links reaches a threshold value. A mean-field Philips-Thorpe rigidity transition line [22] separates these two regions, and has been found close to the $\mathrm{SbTe}_{4}-\mathrm{GeTe}_{4}$ join.

As stated above, the knowledge of the liquid structure can be useful for a first assessment of the amorphous state properties. Another advantage of the liquid phase is that it can be produced in large quantities for any composition of the GeSbTe phase diagram without encountering crystallization. Here, it has to be reminded that the glass-forming region (GFR) of the present ternary is very small and limited to ternary compositions that are close to the eutectic $\mathrm{Ge}_{15} \mathrm{Te}_{85}$ (Fig. 1). Sophisticated techniques such as the twin roller quenching method or thermal coevaporation are needed to extend the amorphous region such as it was shown recently for GeTe and GeGaTe amorphous films [23,24].
In this contribution, we present a combined experimental and computational study on Ge-Sb-Te liquid alloys, which are a useful starting point to describe the amorphous phase at benchmark compositions that are expected to correspond to either flexible, stressed rigid or stress-free compositions. Results from neutron diffraction and inelastic scattering experiments are accompanied with density functional based molecular dynamics simulations including dispersion forces. The latter have been recently shown to give a substantial improvement of the simulated structures $[25,26]$. The combination of experiments and simulations allows a complete description of the structural and vibrational properties of the liquid state, while also providing a neat account for the effect of Te content on structural, vibrational and electronic properties.

The next section will be devoted to the description of the experimental method and computational tools used for this study. Structural properties as illustrated by the structure factors, radial distribution functions, coordination numbers, nature of the neighbors, angular and ring distributions will be given in the third section. The last section will address the issue of vibrational and electronic properties, and the investigation of the vibrational density of states as a function of composition will be detailed. Finally, Sec. V will summarize the main findings and draw some conclusions.

\section{METHODS}

\section{A. Experimental details}

The studied compositions lie on the $\left[\mathrm{Te}-\mathrm{Ge}_{2} \mathrm{Sb}_{2} \mathrm{Te}_{5}\right]$ isopleth and, according to Ref. [15], they were chosen in order to belong either to the flexible phase $\left(\mathrm{Ge}_{5.9} \mathrm{Sb}_{5.9} \mathrm{Te}_{88.2}\right.$ called $\mathrm{Ge}_{6} \mathrm{Sb}_{6} \mathrm{Te}_{88}$ hereafter), or to the stressed-rigid phase $\left(\mathrm{Ge}_{14.3} \mathrm{Sb}_{14.3} \mathrm{Te}_{71.4}\right.$, called $\mathrm{Ge}_{14} \mathrm{Sb}_{14} \mathrm{Te}_{72}$ hereafter). A third composition $\left(\mathrm{Ge}_{10} \mathrm{Sb}_{10} \mathrm{Te}_{80}\right)$ is supposed to be nearly isostatically rigid, as proposed in Ref. [15]. The samples were synthesized from high-purity germanium pellets $(99.999 \%$, Goodfellow), antimony pellets (99.999\%, Sigma-Aldrich) and tellurium ingots (99.9999\%, Sigma-Aldrich). Around $10 \mathrm{~g}$ of stoichiometric powders were placed in a cylindrical silica tube (11 mm inner diameter, $1 \mathrm{~mm}$ thick) further sealed under secondary vacuum $\left(10^{-5} \mathrm{mbar}\right)$. The batch was melted at 1220 $\mathrm{K}$ with a low heating rate of $10 \mathrm{~K} / \mathrm{h}$ and held at this temperature for 3 days. Subsequently, the tubes were cooled down to room temperature with annealing steps of one day at 1173, 1123, and $1073 \mathrm{~K}$. The same samples were used for neutron diffraction and inelastic scattering experiments.

Neutron experiments were performed on the high flux reactor at the Institute Laue Langevin (ILL, Grenoble, France). Diffraction data were acquired on the D4 diffractometer [27]. The incident neutron wavelength was fixed at $0.4976 \AA$ using the $\mathrm{Cu}(220) \mathrm{Bragg}$ reflection. The scattered intensities were measured through an ensemble of nine moving detector banks (each of 64 cells) giving access to a $k=2 \pi \sin 2 \theta$ range of [0.4-23.5] $\AA^{-1}$. The total structure factors $S_{\text {expt }}(k)$ were obtained after standard correction for background and container scattering, self-attenuation, multiple scattering and inelasticity effects. The program CORRECT [28] was used to perform the analysis. Since no liquid density values are available for those compositions, they were first calculated 
from the pure elements assuming zero excess volume of mixing. They were afterward adjusted by using the asymptotic limit of the mean differential scattering cross section per atom that must be equal to the theoretical ones within a few percent. The densities obtained for each composition are given in Table I.

Inelastic neutron scattering experiments were carried on the IN6 spectrometer at a wavelength of $4.14 \AA$. Standard corrections were applied to the data: normalization of the spectra to identical values, subtraction of the container contribution and normalization to a reference spectrum of a $11 \mathrm{~mm}$ diameter vanadium cylinder. The data were corrected for the energy dependent detector efficiency and time independent background. All the correction procedure was done using the LAMP program [29] using predefined functions. A dynamical structure factor $S(2 \theta, \omega)$ was then obtained for each composition. The structure factor $S(k)$ and the VDOS $g(\omega)$ were afterward obtained by integrating $S(2 \theta, \omega)$, respectively, over the accessible $\omega$ range or the accessible $2 \theta$ range.

For both experiments, a vanadium resistor was used as a furnace. The temperature accuracy was estimated using the melting of each alloy, which can be easily identified owing to the disappearance of Bragg peaks. By comparing the obtained melting temperature to the one proposed in Ref. [30], the temperature accuracy was estimated to $\pm 10 \mathrm{~K}$ for the D4 setup and $\pm 30 \mathrm{~K}$ for the IN6 setup. This difference can be explained by the localization of the thermocouples and temperature gradients that are differing from one experiment to the other. The investigated thermodynamic conditions were the same for the two experiments and Table I, summarizes the chosen compositions and temperatures.

\section{B. Molecular dynamics}

The three compositions $\mathrm{Ge}_{x} \mathrm{Sb}_{x} \mathrm{Te}_{100-x}(x=6 \%, 10 \%$, and $14 \%$ ) have been investigated in parallel from molecular dynamics simulations performed in NVT ensemble. In addition, and for completeness, elemental liquid $\mathrm{Te}(x=0)$ and undercooled liquid GST225 $\left(\mathrm{Ge}_{22} \mathrm{Sb}_{22} \mathrm{Te}_{56}, x=22 \%\right)$ were also simulated. Systems contained 300 atoms (Fig. 2) positioned in a periodically repeated cubic cell whose size allows recovering the measured experimental densities $\rho_{\text {expt }}$ reported in Table I. The electronic structure was described within DFT, and evolved self-consistently during the motion [33]. A generalized gradient approximation (GGA) has been used, based on an improved scheme for the exchange-correlation energy obtained by Perdew, Burke, and Ernzerhof (PBEsol). This functional has been found to improve substantially the structural description of elemental tellurium in the liquid phase [34].

Valence electrons were treated explicitly, in conjunction with Trouiller-Martins norm conserving pseudopotentials [35]. The wave functions have been expanded at the $\Gamma$ point of the supercell on a plane wave basis set with an energy cutoff $E_{c}=20 \mathrm{Ry}$. During the Car-Parrinello molecular dynamics (CPMD) simulation, a fictitious electron mass of 2000 a.u. and a time step of $\Delta t=0.12 \mathrm{fs}$ have been used to integrate the equations of motion. Temperature control has been implemented for both the ionic and electronic degrees

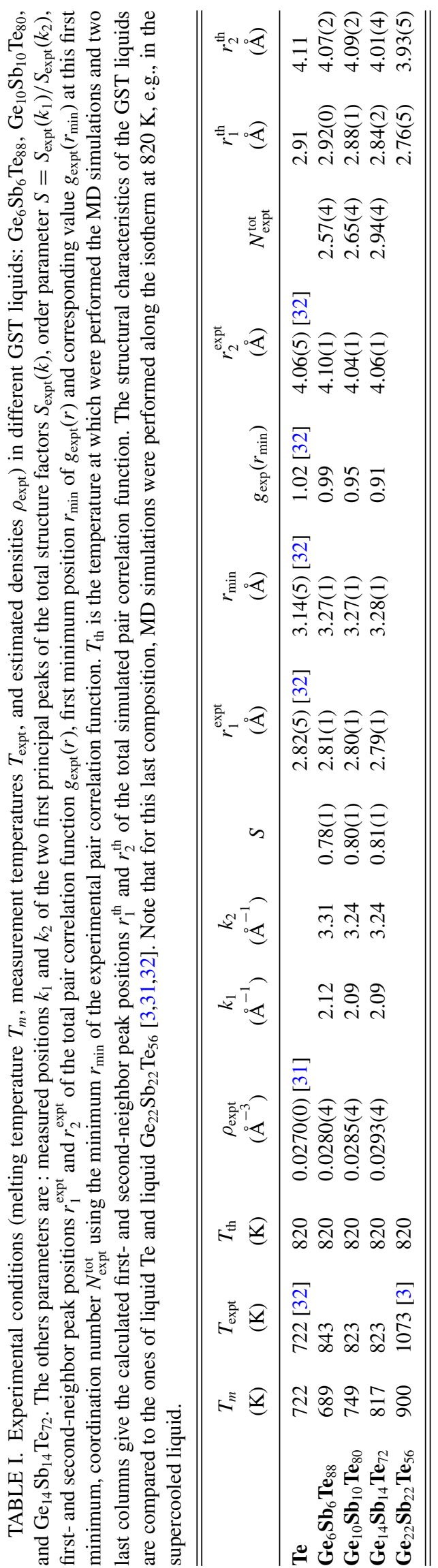




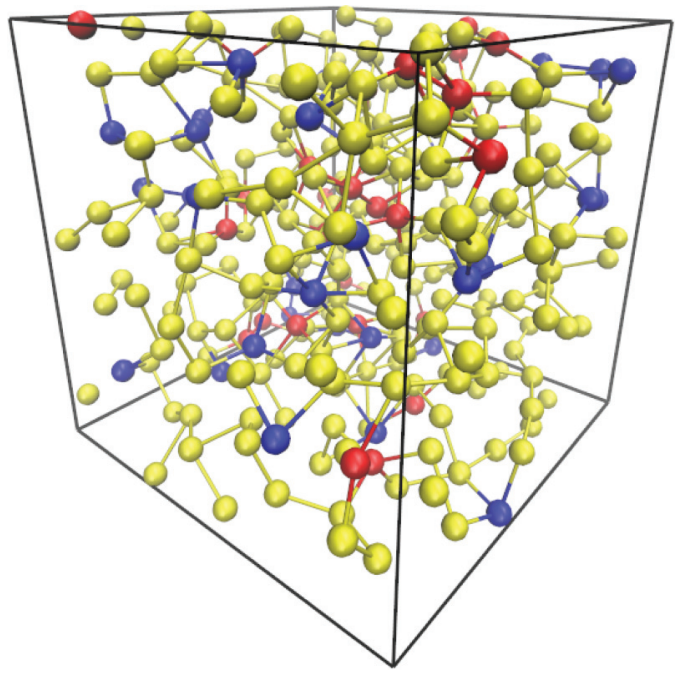

FIG. 2. (Color online) A typical structure of liquid (820 K) $\mathrm{Ge}_{14} \mathrm{Sb}_{14} \mathrm{Te}_{72}$. Ge, Sb, and Te atoms are in red, blue and yellow, respectively.

of freedom by using Nosé-Hoover thermostats [36] with a frequency of $800 \mathrm{~cm}^{-1}$.

In addition, we have used an attractive empirical dispersion coefficient proposed by Grimme [37] :

$$
E_{\mathrm{disp}}=-s_{6} \sum_{i=1}^{N-1} \sum_{j=i+1}^{N} \frac{C_{i j}}{R_{i j}^{6}} f_{\mathrm{dmp}}\left(R_{i j}\right),
$$

where $N$ is the number of atoms of the system, $C_{i j}$ is the dispersion coefficient for atom pair $i j$ and $R_{i j}$ is the interatomic distance. A damping function [37] $f_{\mathrm{dmp}}\left(R_{i j}\right)=$ $\left[1+\exp \left(-d\left(R_{i j} / R_{c}-1\right)\right)\right]^{-1}$ has been also used in order to avoid singularities at short interatomic distances. Here, $R_{c}$ is a cutoff distance canceling $E_{\text {disp }}$ for $R<R_{c}$. Parameters for the Ge-Te systems $\left(R_{c}, C_{i j}, d_{i j}\right)$ are given in Ref. [25], and for the Sb-related pairs, one furthermore has $C_{i j}=397.4$, 361.2, and $265.1 \mathrm{eV} . \AA^{-6}$, and $R_{c}=3.76,3.61$, and $3.77 \AA$ for Sb-Sb, Sb$\mathrm{Te}$, and $\mathrm{Sb}-\mathrm{Ge}$, respectively [37]. For comparison, simulations without the dispersion forces have been performed under the same thermodynamic conditions for selected situations, and will serve in the discussion throughout the paper.

The starting configuration of all liquids is a random structure of $\mathrm{Ge}, \mathrm{Sb}$, and Te atoms fulfilling the three experimentally investigated stoichiometries $(6 \%, 10 \%$, and $14 \%)$. Loss of the memory of the initial configurations has been achieved through preliminary runs at 2000 and $1500 \mathrm{~K}$ over 50 ps at densities corresponding to a high temperature extrapolation of the experimental densities. The total simulation time of these preliminary runs induces mean square displacements of the order of several cell lengths, which provides a good indication that the memory of the initial configurations has been lost. After equilibrating at different target temperatures corresponding to the experimental investigated temperatures over $5 \mathrm{ps}$, trajectories for each compositions have been accumulated over 25-30 ps each and used for the analysis of structural and vibrational properties. The obtained structure (e.g., Fig. 2) reveals the presence of various structural motifs

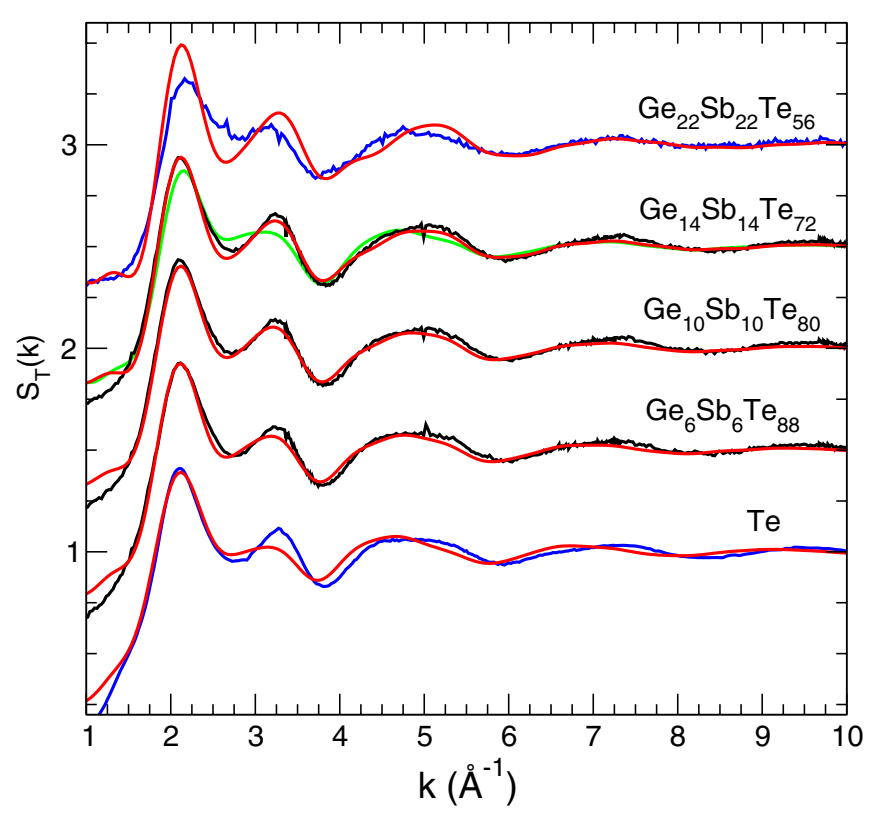

FIG. 3. (Color online) Evolution of the simulated total structure factor $S_{\mathrm{th}}(k)$ (red curves) with composition $x$ along the isotherm $T=$ $820 \pm 20 \mathrm{~K}$ in $\mathrm{Ge}_{x} \mathrm{Sb}_{x} \mathrm{Te}_{100-2 x}$ liquids, compared to the experimental counterpart $S_{\text {expt }}(k)$ (black curves). From bottom to top, $x=0 \%, 6 \%$, $10 \%, 14 \%$, and $22 \%$. The green curve is a simulation of $\mathrm{Ge}_{14} \mathrm{Sb}_{14} \mathrm{Te}_{72}$ without the dispersion correction. Note that experimental temperature for elemental liquid Te was equal to $763 \mathrm{~K}$ [32] and to $1073 \mathrm{~K}$ for liquid $\mathrm{Ge}_{22} \mathrm{Sb}_{22} \mathrm{Te}_{56}$ [3] (blue curves), while MD simulations were performed at $820 \mathrm{~K}$, i.e., in the supercooled liquid for $\mathrm{Ge}_{22} \mathrm{Sb}_{22} \mathrm{Te}_{56}$.

such as pyramidal local structures for $\mathrm{Ge}$ or $\mathrm{Sb}$, whereas tellurium chain fragments are also visible. With the simulation boxes using $\rho_{\text {expt }}$, the residual pressures at all temperatures were found to be of about $0.2-0.4 \mathrm{GPa}$.

\section{STRUCTURAL PROPERTIES}

The total experimental structure factors $S_{\text {expt }}(k)$ and their corresponding total pair correlation functions $g_{\text {expt }}(r)$ are respectively presented in Figs. 3 and 4 together with the results from the MD simulations $g_{\mathrm{th}}(r)$ and $S_{\mathrm{th}}(k)$.

\section{A. Qualitative analysis of the experimental data}

In order to evidence some trends in the experimental data, the order parameter $S=S\left(k_{2}\right) / S\left(k_{1}\right)$ has been calculated. As previously proposed in Refs. [3,26], this parameter may be useful to distinguish between octahedral $(S<1)$ and tetrahedral $(S>1)$ liquids. Indeed, it has been proposed that tetrahedral liquids (such as silica [38] or $\mathrm{GeSe}_{2}$ [39]) display a proeminent secondary peak at $k=k_{2}$ that is more intense than the first peak (at $k_{1}$ ) of the structure factor $S(k)$. The opposite behavior is found for octahedral liquids (e.g., SnTe [3]). Values of this parameter are given in Table I for the present system. For all compositions, $S$ values are below 1 showing that the liquids display a tendency towards octahedral local order.

In Ref. [26], we have found for the liquid $\mathrm{Ge}_{20} \mathrm{Te}_{80}$ that $S$ was equal to $0.83-0.86$ over the temperature range 700-923 K. From the obtained values reported in Table I for the $10 \%$ composition (i.e., the composition having an 


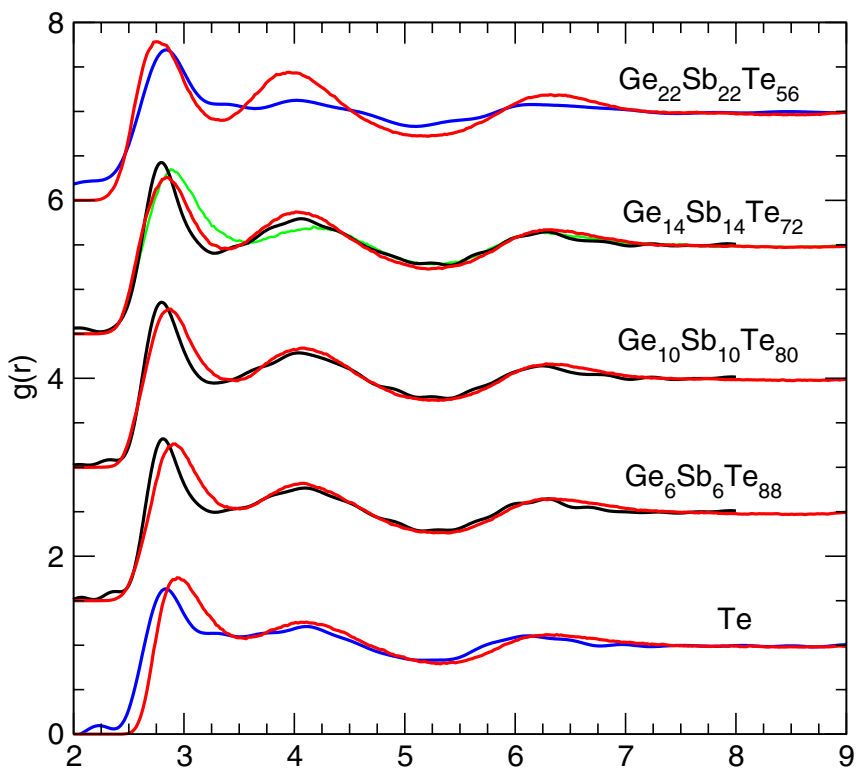

FIG. 4. (Color online) Evolution of the simulated pair correlation function $g_{\text {th }}(r)$ with composition $x$ along the isotherm $T=820 \pm$ $20 \mathrm{~K}$ in $\mathrm{Ge}_{x} \mathrm{Sb}_{x} \mathrm{Te}_{100-2 x}$ liquids (red curves, compared to the experimental counterpart $g_{\text {expt }}(r)$ (black curves). From bottom to top, $x=0 \%, 6 \%, 10 \%, 14 \%$, and $22 \%$. The green curve represents results from a simulation without the Grimme correction for $x=14 \%$. Note that experimental temperature for elemental liquid Te was equal to $763 \mathrm{~K}$ [32] and to $1073 \mathrm{~K}$ for liquid $\mathrm{Ge}_{22} \mathrm{Sb}_{22} \mathrm{Te}_{56}$ [3] (blue curves) while MD simulations were performed at $820 \mathrm{~K}$.

equivalent amount of Te atoms as $\mathrm{Ge}_{20} \mathrm{Te}_{80}$ ), we can conclude that the substitution of germanium by antimony decreases $S$, and, therefore, may indicate a stabilization of the octahedral character of the melts.

The total pair correlation functions $g_{\text {expt }}(r)$ are presented in Fig. 4. While the first distance does not change within the experimental error, the second distance seems to lightly decreases when decreasing Te content, which may indicates that with increasing $\mathrm{Sb} / \mathrm{Ge}$ content the local structure is more compact.

Using the total pair correlation functions $g_{\text {expt }}(r)$ and the experimentally estimated densities $\rho_{\text {expt }}$ (see Table I), the total coordination numbers

$$
N_{\mathrm{expt}}^{\mathrm{tot}}=4 \pi \rho_{\mathrm{expt}} \int_{r_{0}}^{r_{\mathrm{min}}} r^{2} g_{\text {expt }}(r) d r
$$

can be obtained as a function of Te content. $N_{\text {expt }}^{\text {tot }}$ values are listed in Table I, together with the value of the upper integration bound $r_{\text {min }}$. The lower integration bound $r_{0}$ is fixed to $2.20 \AA$ for all the thermodynamic states. It is noticeable that, along the isotherm at $(823 \pm 20 \mathrm{~K})$, the total coordination number increases with increasing $\mathrm{Sb} / \mathrm{Ge}$ content, which indicates that the liquid network structure increases its average connectivity.

\section{B. Structural analysis using MD simulation}

\section{Comparison with experiments}

In Fig, 3, the experimental structure factors are compared with the total calculated structure factors $S_{\mathrm{th}}(k)$ defined by

$$
S_{\mathrm{th}}(k)=\frac{\sum_{i, j} c_{i} c_{j} b_{i} b_{j} S_{i j}(k)}{\left(\sum_{i, j} c_{i} b_{i}\right)^{2}},
$$

where the concentrations $c_{i}(i=\mathrm{Ge}, \mathrm{Sb}$, and $\mathrm{Te})$ depend on the considered stoichiometry, and $b_{i}$ are the neutron scattering lengths given by $b_{\mathrm{Ge}}=8.185 \mathrm{fm}, b_{\mathrm{Sb}}=5.57 \mathrm{fm}$, and $b_{\mathrm{Te}}=$ $5.68 \mathrm{fm}$, respectively [40].

It can be remarked that the experimental data of $S_{\text {expt }}(k)$ are very well reproduced over the entire range of wave vectors, this statement being valid for all compositions. When followed as a function of composition, we furthermore note that the agreement with the experimental function seems to increase with modifier composition, i.e., as one moves from $\mathrm{Ge}_{6} \mathrm{Sb}_{6} \mathrm{Te}_{86}$ to $\mathrm{Ge}_{14} \mathrm{Sb}_{14} \mathrm{Te}_{72}$.

As the simulation without dispersion forces is considered (green curve in Fig. 3), one furthermore realizes that taking into account Eq. (1) leads to a clear improvement of the calculated structure factor $S_{\mathrm{th}}(k)$ when compared to the experimental counterpart $S_{\text {expt }}(k)$. We observe, indeed, that the lack of dispersion forces leads to a secondary peak at $k=k_{2}$ that is less well defined, and whose intensity is substantially reduced when compared to the experimental function. In addition, the absence of dispersion forces leads to a redshift of the peak at $\simeq 5.5 \AA^{-1}$.

With the important discrepancies found between $k=2.5$ and $5 \AA^{-1}$ in the reproduction of $S(k)$ without taking into account Eq. (1), one may argue at a first stage that the impact of the dispersion correction in real space must be particularly active at distances $7.7 / k$ found between $\simeq 3$ and $1.5 \AA$ [41]. However, the effect appears to be more subtle. In fact, the Grimme correction is only active for distances larger than $r>$ $R_{c}$ or for wave vectors $k<7.7 / R_{c}$ [41]. With the values used for $R_{c}(\simeq 3.5 \AA$ [25]), this means that Eq. (1) should influence the structure in reciprocal space only for $k<2.2 \AA^{-1}$, i.e., at wave vectors for which the agreement with experiments from both simulations [with or without Eq. (1)] is satisfied.

However, it should be reminded that for simple LennardJones systems, the low- $k$ oscillations in the structure factor arise from the attractive part of the interaction [42], and lead to an increased intensity of the principal peak when compared to a hard sphere liquid. Here, the better resolved peaks (i.e., more pronounced oscillations) in $S_{\mathrm{th}}(k)$ for $\mathrm{Ge}_{14} \mathrm{Sb}_{14} \mathrm{Te}_{72}$ (Fig. 3) using the Grimme correction can therefore be considered as a direct result of the presence of these additional attractive forces. These forces such as those provided by Eq. (1), besides determining the thermodynamic properties of the liquid and modifying the oscillations of the structure factor, usually lead to even more important changes in the very low wave-vector region [43], and particularly to the rising [44,45] of $S(k)$ in the limit $k \rightarrow 0$, which reflects the effect on the liquid compressibility $\kappa_{T}$ given that $S(0)=\rho \mathrm{k}_{B} T \kappa_{T}$. However, this momentum transfer region is obviously out of reach given our finite simulation cell.

The same conclusions regarding the agreement with experiments remain valid in real space. As seen in Fig. 4, a very good agreement is obtained between the simulated and experimental pair correlation function, the peak positions and 
intensities being merely reproduced at all distances. Such main peak positions of the simulated $g(r)\left(r_{1}^{\text {th }}\right.$ and $r_{2}^{\text {th }}$, see Table I) can be compared to experimental findings. It is seen that while the first peak position (i.e., the bond distance at $r_{1}^{\text {th }}$ ) shows a small difference with the experimental determination $r_{1}^{\text {expt }}$, the agreement is excellent for the secondary peak found at $r_{2}^{\text {th }}$. This statement is true for all compositions, all also directly observed from Fig. 4.

As noticed when the simulated $g(r)$ of $\mathrm{Ge}_{14} \mathrm{Sb}_{14} \mathrm{Te}_{72}$ and $\mathrm{Ge}_{6} \mathrm{Sb}_{6} \mathrm{Te}_{88}$ are compared, the agreement between simulated and experimental pair correlation function tends to improve as the concentration $x$ is increased. This trend can be linked to the increasing effect of the dispersion forces which are enhanced with increasing Ge composition. In fact, the polarizability is affected by the increased presence of heteropolar Ge-Te and $\mathrm{Sb}$-Te bonds, which involve atoms having different electronegativities $(2.10,2.08$, and 2.01 for $\mathrm{Te}, \mathrm{Sb}$ and $\mathrm{Ge}$ on the Pauling scale [46], respectively). This in turn gives rise to the possibility of having an increased number of dipolar interactions and an increased effect due to such dispersion forces. When such forces are neglected (e.g., green curve Fig. 4), one recovers the usual bond distance problem encountered in DFT simulations without Grimme corrections. In this latter situation, both first and second peak positions are overestimated, i.e., one has $r_{1}^{\text {th }}=2.88 \AA$ $\left(r_{1}^{\text {expt }}=2.79 \AA\right)$ and $r_{2}^{\text {th }}=4.17 \AA\left(r_{2}^{\text {expt }}=4.01 \AA\right)$. Moreover, with a global shift of the first peak and a minimum position $r_{\text {min }}$, a simulation without the dispersion correction of Eq. (1) will automatically [Eq. (2)] lead to an overestimation of the coordination numbers, as discussed below.

\section{Effect of composition}

The effect of composition can be tracked from the evolution of both the total structure factor (Fig. 3) and the pair correlation function (Fig. 4). Both principal peaks (PPs) are found to slightly vary with composition, e.g., the first peak shifts to higher $k$ between $10 \%\left(2.13 \AA^{-1}\right)$ and $14 \%$ Ge $\left(2.18 \AA^{-1}\right)$, a result that can not be attributed to the change in liquid density alone (Table I) and is therefore linked to the occurrence of smaller long-range correlations as the density of Ge increases.

In real space, it can be observed that the changes in composition mostly affects the secondary peak at $r=r_{2}$ as already observed in the experimental data. Similarly, the total coordination number $N_{\text {th }}^{\text {tot }}$ calculated from the area of the first peak in the total pair correlation function (Fig. 4) using Eq. (2) leads to values that are close to those determined experimentally (Table I), and both total running coordination numbers $N_{\text {th }}^{\text {tot }}(r)$ and $N_{\text {expt }}^{\text {tot }}(r)$ are close for nearly all compositions. The differences emerging for the total coordination number thus only arise from the choice of the minimum $r_{\text {min }}$. For instance, we find $N_{\text {th }}^{\text {tot }}=3.04\left(r_{\min }=3.33 \AA\right)$ for $\mathrm{Ge}_{10} \mathrm{Sb}_{10} \mathrm{Te}_{80}$ (experimentally $N_{\text {th }}^{\text {tot }}=2.65$, see Table I). This value reduces to 2.68 if the same cutoff $r_{\min }$ is used $(3.27 \AA$ ). For $\mathrm{Ge}_{14} \mathrm{Sb}_{14} \mathrm{Te}_{72}$, one has $N_{\text {th }}^{\text {tot }}=3.39\left(r_{\text {min }}=3.30 \AA\right)$, which is much smaller than the value $N_{\text {th }}^{\text {tot }}=4.72\left(r_{\min }=3.55 \AA\right)$ obtained without the Grimme correction (from the green curve in Fig. 4).

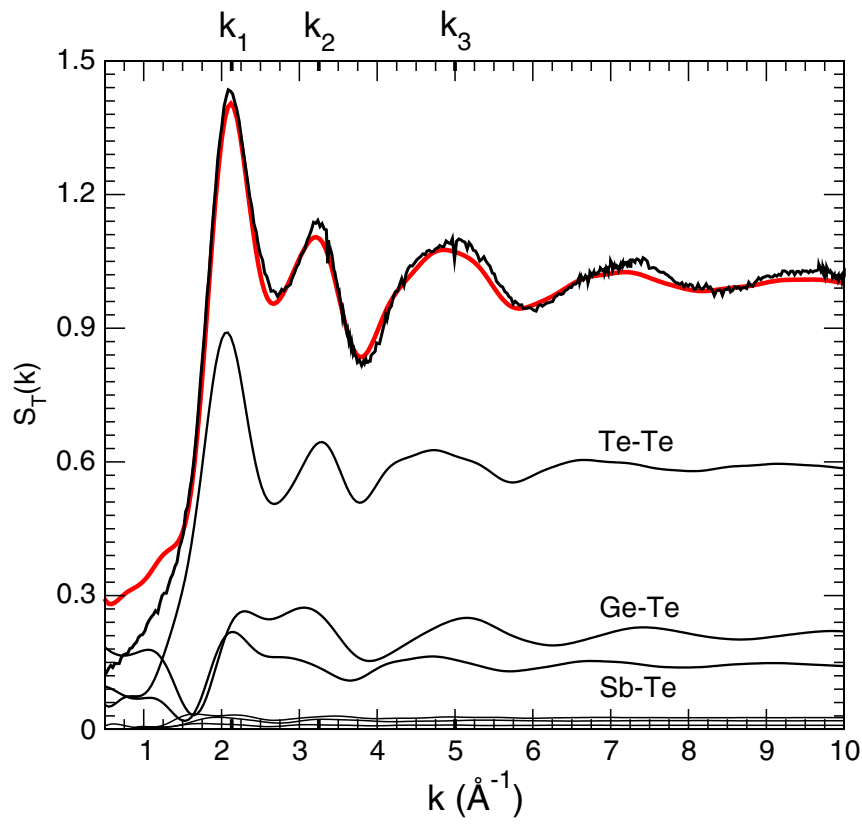

FIG. 5. (Color online) A typical decomposition of the total simulated structure factor $S_{\text {th }}(k)$ of liquid $(820 \mathrm{~K}) \mathrm{Ge}_{10} \mathrm{Sb}_{10} \mathrm{Te}_{80}$ (same as Fig. 3) into partial structure factor $\left(2-\delta_{i j}\right) c_{i} c_{j} b_{i} b_{j} S_{i j}(k) /\langle b\rangle^{2}$. Note that the black lines close to $S(k) \simeq 0$ correspond to the remaining pairs (Ge-Ge, Ge-Sb, and $\mathrm{Sb}-\mathrm{Sb})$. The position of the main peaks $\left(k_{1}\right.$, $\left.k_{2}, k_{3}\right)$ are indicated on the top axis.

\section{Partial contributions}

\section{Reciprocal space}

For all compositions, total structure factors $S(k)$ are dominated by three pairs (Ge-Te, Sb-Te, and Te-Te). Figure 5 shows, as an example, the contributions of such pairs to the simulated $S_{\mathrm{th}}(k)$ in the $\mathrm{Ge}_{10} \mathrm{Sb}_{10} \mathrm{Te}_{80}$ liquid. All other pairs are found to contribute to less than $\simeq 3 \%$, given the chosen interval in composition $(0 \% \leqslant x \leqslant 22 \%)$, and their neutron coherent lengths $b_{i}$.

From Fig. 5, one furthermore detects which pair contributes to the observed peaks of the total measured or simulated structure factor (Fig. 3). It is found that $S_{\mathrm{TeTe}}(k)$ contributes to all relevant peaks, i.e., first and second peaks at $k_{1}=2.13 \AA^{-1}$ and $k_{2}=3.25 \AA^{-1}$, but also to the third broad peak found at $k_{3} \simeq 5 \AA^{-1}$. On the other hand, the two other relevant partials, $\mathrm{Ge}-\mathrm{Te}$ and $\mathrm{Sb}-\mathrm{Te}$, have mostly contributions at $k_{1}$ and $k_{2}$.

Such partial structure factors $S_{i j}(k)$ can be studied as a function of composition (Fig. 6), and one can remark that the most important changes are found in the Te-Te partial. Indeed, for low $x$ neither Ge-Te, nor Sb-Te show significant changes in structural correlations at intermediate range order distances given the nearly absence of variation of $S_{\mathrm{GeTe}}(k)$ and $S_{\mathrm{SbTe}}(k)$ with Te content. Still, it can be noticed that in the low momentum transfer limit $\left(k<2 \AA^{-1}\right)$, a small variation of the Ge-Te negative oscillation features corresponding to structural variations at long range distances, the evolution being particularly visible as the content $x$ is increased from $14 \%$ to $22 \%$ (GST225). 

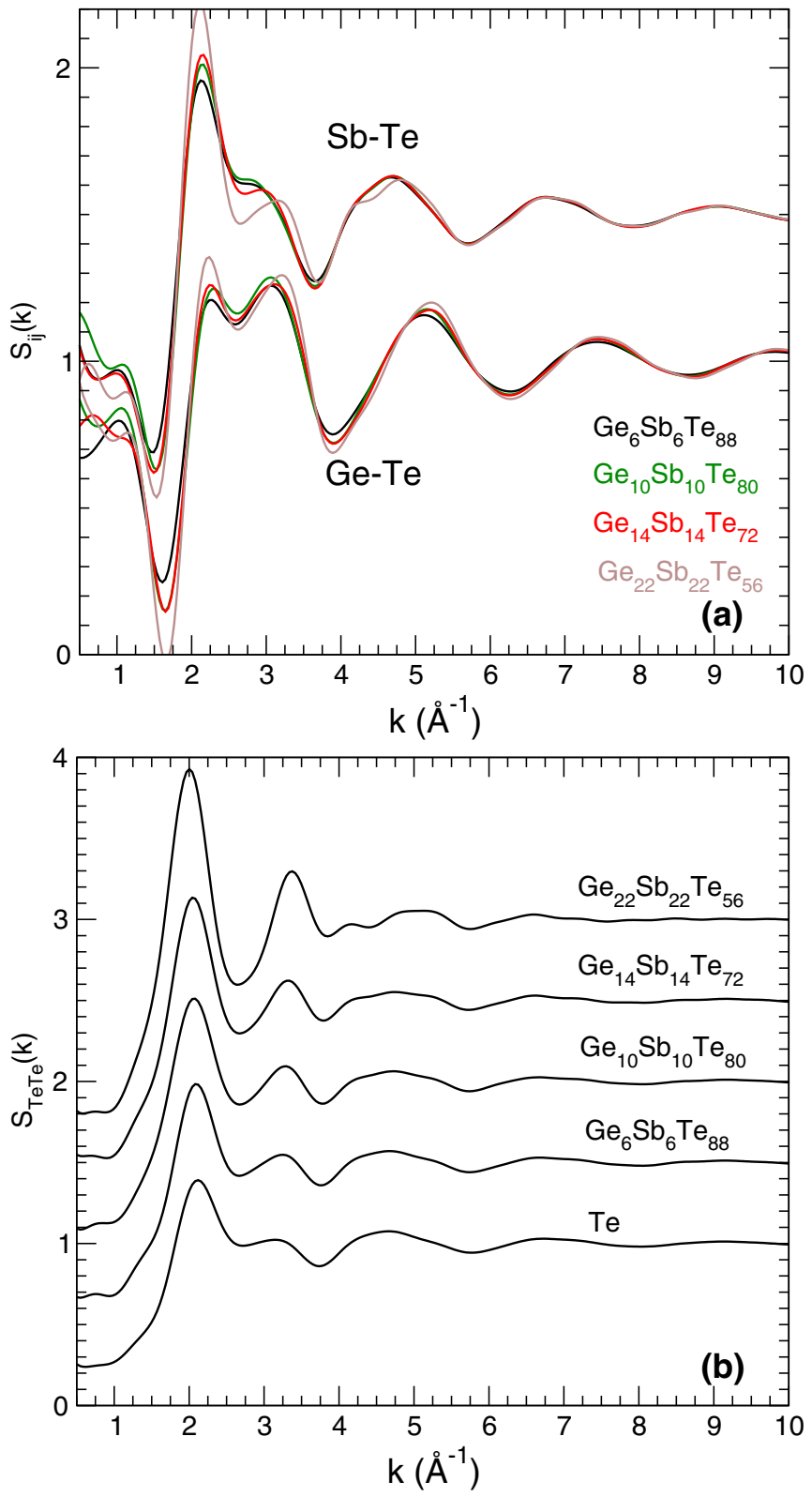

FIG. 6. (Color online) Evolution of the simulated partial structure factor $S_{i j}(k)$ with composition $x$ in $\mathrm{Ge}_{x} \mathrm{Sb}_{x} \mathrm{Te}_{100-2 x}$ liquids at $820 \mathrm{~K}$. (a) Ge-Te and Sb-Te partials and (b) Te-Te partials at $820 \mathrm{~K}$.

In addition, we must stress that both partials Ge-Te and $\mathrm{Sb}$-Te do not have at all the same shape because $S_{\mathrm{SbTe}}$ has a strong peak at $k=k_{1}$ but is still weakly contributing to the total $S_{\mathrm{th}}(k)$, especially when it is compared to the contribution of Te-Te (see Fig. 5). On the other hand, $S_{\mathrm{GeTe}}$ displays two peaks at $k \simeq k_{1}$ and $k \simeq k_{2}$ with the same intensity. One can thus, argue, that structural correlations induced by $\mathrm{Ge}$ or $\mathrm{Sb}$ cross-linking are not equivalent and affect structural correlations at $k<4 \AA^{-1}$ in a different way. Finally, it can be noticed that the GST225 undercooled liquid is certainly different from the other alloys having a larger Te content. Both $\mathrm{Sb}$-Te and Ge-Te partial structure factors are found to display increased oscillations at $2 \leqslant k \leqslant 4 \AA^{-1}$ when compared to counterparts at higher Te content. For instance, the position of the shoulder peak found at $\simeq 2.8 \AA^{-1}$ in the function $S_{\mathrm{SbTe}}(k)$ is found to increase substantially between the $14 \%$ and $22 \%$ liquid, and leads to a separate peak at $3.2 \AA^{-1}$ in GST225 that also contribute to the growth of the corresponding peak in the total $S_{\mathrm{th}}(k)$ (see Fig. 3). Similarly, we find that the FSDP and the first peak intensity of $S_{\mathrm{GeTe}}(k)$ markedly increases between these two compositions, but most of the changes appearing in the total structure factor (Fig. 3) arise from those found in the Te-Te partial structure factor [Fig. 6(b)].

\section{Real space}

The analysis of the structure in real space can be characterized from the partial pair correlation functions, as shown in Fig. 7. A certain number of features should be emphasized. First, we note that Ge-Ge and Sb-Sb homopolar bonds exist for all compositions, including for the one with the highest Te content $\mathrm{Ge}_{6} \mathrm{Sb}_{6} \mathrm{Te}_{88}$, the former leading to a peak in the partial pair correlation function $g_{\mathrm{GeGe}}(r)$ at $2.61 \AA$, while the latter exhibits a peak at $3.05 \AA$ in $g_{\mathrm{SbSb}}(r)$. However, this contribution is very small and an inspection of the atomic snapshots indicates that a single Ge-Ge bond is involved in the structure. Furthermore, when the statistics of bonds is computed (Fig. 8), it is seen that this fraction is negligible compared to the other bond populations (Ge-Te, Te-Te, and $\mathrm{Sb}-\mathrm{Te}$ ), which dominate the structure in the Te-rich liquids, the fraction of $\mathrm{Ge}-\mathrm{Ge}$ and $\mathrm{Sb}-\mathrm{Sb}$ starting to increase only for $x>10 \%$, a feature also noticed in a similar study on amorphous Ge-Te [23].

With increasing Ge/Sb content, such homopolar distances tend to reduce given that $d_{\mathrm{GeGe}}$ reduces from 2.61 to $2.51 \AA$ for the $22 \%$ supercooled liquid, whereas $d_{\mathrm{SbSb}}$ decreases to a lesser extent from 3.05 to $3.01 \AA$ under the same increase in $x$. Such reductions seem to parallel the evolution of the density $\rho_{\text {expt }}$ along the $\simeq 820 \mathrm{~K}$ isotherm, which increases by about $\simeq 5 \%$ (from 0.0280 to $0.293 \AA^{-3}$ ).

\section{Coordination numbers}

In Table II, we report the partial pair coordinations $n_{i j}$ calculated from the partial pair correlation functions (Fig. 7). It is seen that with increasing $\mathrm{Ge} / \mathrm{Sb}$ content, the partial coordination numbers $n_{\mathrm{GeTe}}$ and $n_{\mathrm{SbTe}}$ decrease with increasing $x$, the latter being always larger (4.53-5.12) than $n_{\mathrm{GeTe}}(3.75-$ 4.21), and exhibiting important decrease between $14 \%$ and $22 \%$. This decrease is related with the growth of homopolar coordination number $n_{\mathrm{SbSb}}$, and a similar trend with $x$ is obtained for $n_{\mathrm{GeGe}}$.

Simulations without the Grimme corrections will lead to larger coordination numbers because the corresponding minima $r_{i j}$ of the partial pair correlation functions $g_{i j}(r)$ are found at larger distances, which result from a shift of the main peak (inset GeTe of Fig. 7). Here, it is seen that for, e.g., $n_{\mathrm{SbTe}}$, one finds 5.43 [against 4.53 using Eq. (1)] or $n_{\mathrm{GeTe}}=4.03$ (against 3.75).

Corresponding total coordination numbers can then be calculated (Table III) using

$$
n_{i}=n_{i i}+\sum_{i \neq j} n_{i j},
$$



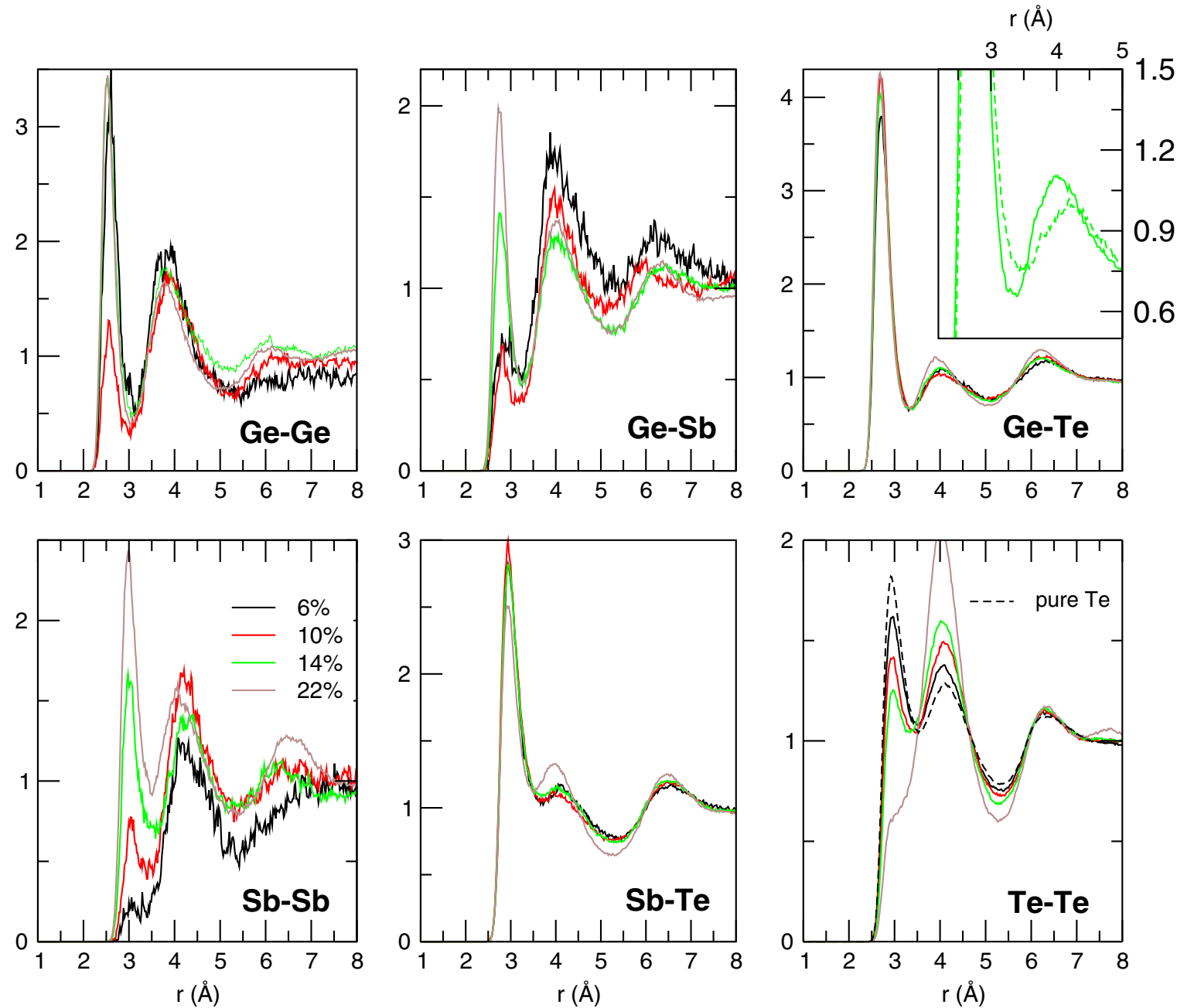

FIG. 7. (Color online) Evolution of the simulated partial pair correlation functions $\mathrm{g}_{i j}(r)$ at fixed temperature $(820 \pm 20 \mathrm{~K})$ as a function of composition $x$ in $\mathrm{Ge}_{x} \mathrm{Sb}_{x} \mathrm{Te}_{100-2 x}$ liquids. The inset in the GeTe panel shows a direct comparison of the effect of the correction of Eq. (1) on $g_{\mathrm{GeTe}}(r)$ for the $\mathrm{Ge}_{14} \mathrm{Sb}_{14} \mathrm{Te}_{72}$ liquid: solid green curves (same as main panel), broken green curve (simulation without dispersion forces).

and, e.g., $(1-2 x) n_{\mathrm{TeGe}}=x n_{\mathrm{GeTe}}$. Results show that the atoms do not follow the $8-N$ rule ( $\mathrm{N}$ being the number of $p$ and $s$ electrons). One finds, indeed, that $\mathrm{Ge}$ atoms have a coordination number of about 4.13-4.40, whereas $\mathrm{Sb}$ atoms have a slightly larger coordination number $\left(n_{\mathrm{Sb}}=4.93-5.48\right)$. This arises from the fact that the dominant geometry of $\mathrm{Sb}$ is octahedral (see below) with vacancies given that $n_{\mathrm{Sb}}<6$, whereas $\mathrm{Ge}$ atoms can be found in two local geometries, defect octahedral and tetrahedral [18]. Te atoms have a coordination number that is always larger than three, i.e., one finds $n_{\mathrm{Te}}=3.61-3.93$ for the different compositions, and both $\mathrm{Ge}_{10} \mathrm{Sb}_{10} \mathrm{Te}_{80}$ and $\mathrm{Ge}_{14} \mathrm{Sb}_{14} \mathrm{Te}_{72}$ seem to display increased Te coordination numbers (3.90-3.93), a tendency that is barely observable from the partials (Fig. 7) but may be reminiscent of the increased liquid density.

In order to be comparable with previous findings, we have calculated the coordination numbers $n_{\mathrm{Ge}}, n_{\mathrm{Sb}}$, and $n_{\mathrm{Te}}$ for GST225 at the same cut-off distance $r_{i j}^{m}=3.2 \AA$ as in Ref. [9], and corresponding results are shown in the bottom of Table III. It is seen that the incorporation of a Grimme correction systematically lowers the coordination numbers for this composition, a result that can be, again, already anticipated from the inspection of Fig. 4, which shows a shift of the first peak and first minimum to higher distances $r$. Furthermore, as seen from Table II, the cutoff distance of $3.2 \AA$ lies well below the cutoff distances $r_{i j}^{m}$ determined at the minimum of the partial pair correlation functions $g_{i j}(r)$ (Fig. 7) or even from those determined from the species related correlation functions $g_{i}(r)$ (Table III). Recently, we have shown [26] that the coordination numbers are highly sensitive to the choice made for $r_{i j}^{m}$, and the present results confirms this property for the case of $\mathrm{Ge}-\mathrm{Sb}$-Te liquids.

\section{E. Bond angles}

Figures 9 and 10 display bond angle distributions present in the liquid structures for the different compositions. It is found that the effect of composition is barely observed on the bond angle distributions involving essentially heteropolar bondings (Fig. 9) such as Ge-Te-Ge or Te-Ge-Te, and particularly Te-Sb-Te, which shows virtually no changes over the whole compositional interval of interest [Fig. 9(d)]. However, at low Te content, the only visible changes are found for the $6 \%$ composition for Sb-Te-Sb bond angles [Fig. 9(b)], which 


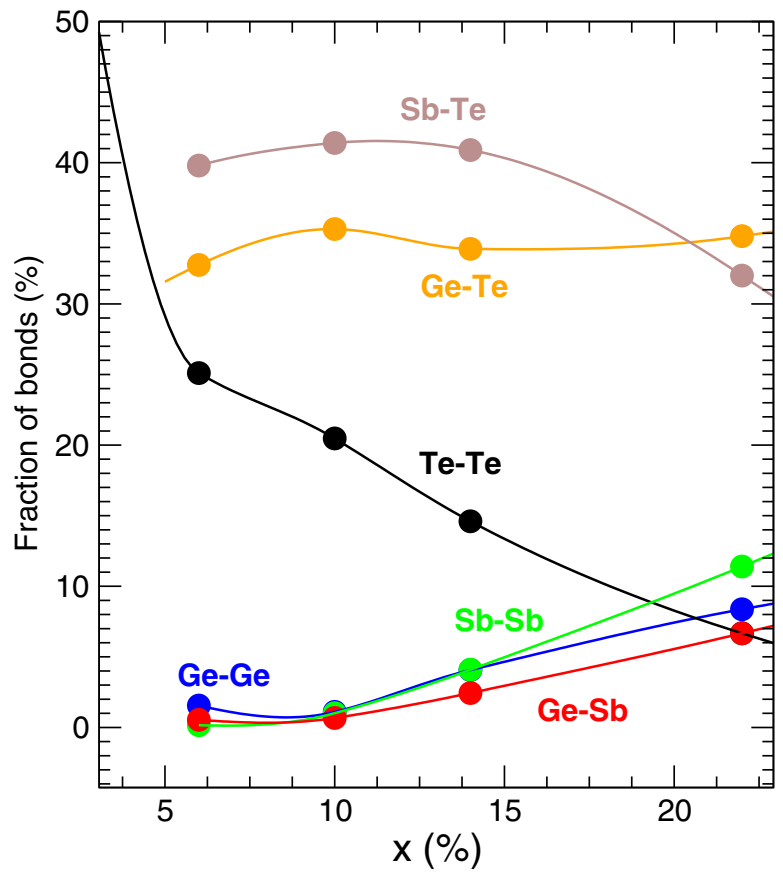

FIG. 8. (Color online) Calculated fraction of bonds in liquid $(820 \mathrm{~K}) \mathrm{Ge}-\mathrm{Sb}-\mathrm{Te}$ liquids as a function of modifier concentration $x$.

show an increased contribution at $\simeq 80^{\circ}-90^{\circ}$. For the largest composition $\left(\mathrm{Ge}_{22} \mathrm{Sb}_{22} \mathrm{Te}_{56}\right)$ an increased tendency to form Ge octahedral geometries is detected, which manifests by a growth of the contribution at $\simeq 90^{\circ}$ and $\simeq 160^{\circ}$ for Te-Ge-Te [Fig. 9(c)]. The variation in the Te-Te-Te bond angle distribution [Fig. 9(e)] between the $14 \%$ and the $22 \%$ composition indicates that the remaining Te-Te-Te fragments are found in an increased chain structure due to the noticeable growth of the $160^{\circ}$ contribution, but this may also arise from the poor statistics of such structural motifs at elevated $\mathrm{Ge} / \mathrm{Sb}$ content. For larger Te content, the Te-Te-Te bond angle distribution is found to be extremely close to the one calculated for elemental Te $[x=0 \%$, orange curve in Fig. 9(e)]. This indicates that the angles involved in such Te-Te-Te fragments are weakly affected by the $\mathrm{Ge} / \mathrm{Sb}$ induced crosslinking of the structure.

The increased coordination number of $\mathrm{Sb}$ atoms (Table III, as compared to $\mathrm{Ge}$ ) is also consistent with the shape of the Te-Sb-Te and Te-Ge-Te bond angle distributions. The former displays indeed a well defined contribution around $90^{\circ}$ indicative of defect octahedral geometries, whereas the latter has a broader distribution that signals the presence of mixed geometries, defect octahedra and tetrahedra.

Similar results are found for bond angle distributions with a homopolar bond [Figs. 10(a)-10(d)] involving a Ge atom (Te$\mathrm{Te}-\mathrm{Ge}, \mathrm{Ge}-\mathrm{Ge}-\mathrm{Te}$ ) or a $\mathrm{Sb}$ atom (Te-Te-Sb and $\mathrm{Sb}-\mathrm{Sb}-\mathrm{Te}$ ), all of them (except $\mathrm{Sb}-\mathrm{Sb}-\mathrm{Te}$ ) displaying the same contributions at $\simeq 90^{\circ}$ and $160^{\circ}$. Both Te-Te-Ge [Fig. 10(a)] and Te-Te-Sb [Fig. 10(b)] have an additional contribution at $\simeq 60^{\circ}$ that we identify from a visual inspection of the atomic structure with angles defined by three atoms found within the equatorial plane of a Te-centred defective octahedra. A similar contribution at $\simeq 60^{\circ}$ in the Ge-Ge-Te bond angle distribution [Fig. 10(c)] signals the presence of edge-sharing structures (4-membered rings, see below), and this contribution is enhanced for the
TABLE II. Cutoff distance $r_{i j}^{m}$ (minimum of the corresponding function $\left.g_{i j}(r)\right)$, calculated partial coordination numbers $n_{i j}=$ $n_{i j}\left(r_{m}\right)$ with $i, j=(\mathrm{Ge}, \mathrm{Sb}, \mathrm{Te})$ at different compositions $x$ in the $(820 \pm 20 \mathrm{~K}) \mathrm{Ge}_{x} \mathrm{Sb}_{x} \mathrm{Te}_{100-2 x}$ liquid. Calculations for $\mathrm{Ge}_{14} \mathrm{Sb}_{14} \mathrm{Te}_{72}$ liquid are performed with or without the dispersion correction [Eq. (1)].

\begin{tabular}{lccccccc}
\hline \hline System & & $n_{\mathrm{GeGe}}$ & $n_{\mathrm{GeTe}}$ & $n_{\mathrm{TeTe}}$ & $n_{\mathrm{SbSb}}$ & $n_{\mathrm{SbTe}}$ & $n_{\mathrm{GeSb}}$ \\
\hline $\mathbf{G e}_{6} \mathbf{S b}_{6} \mathbf{T e}_{88}$ & $\left.r_{i j}^{m}(\AA)\right)$ & 3.15 & 3.34 & 3.52 & 3.39 & 3.69 & 3.23 \\
& & 0.20 & 4.21 & 3.23 & 0.02 & 5.12 & 0.07 \\
$\mathbf{G e}_{10} \mathbf{S b}_{10} \mathbf{T e}_{80}$ & $r_{i j}^{m}(\AA)$ & 3.03 & 3.34 & 3.45 & 3.42 & 3.69 & 3.18 \\
& & 0.13 & 4.19 & 2.43 & 0.12 & 4.92 & 0.08 \\
$\mathbf{G e}_{14} \mathbf{S b}_{14} \mathbf{T e}_{72}$ & $r_{i j}^{m}(\AA)$ & 3.06 & 3.34 & 3.30 & 3.56 & 3.69 & 3.24 \\
& & 0.45 & 3.75 & 1.62 & 0.44 & 4.53 & 0.27 \\
$\mathbf{G e}_{14} \mathbf{S b}_{14} \mathbf{T e}_{72}$ & $r_{i j}^{m}(\AA)$ & 3.10 & 3.47 & 3.58 & 3.54 & 3.86 & 3.33 \\
without Eq. $\left._{1}\right)$ & & 0.70 & 4.03 & 2.86 & 0.41 & 5.43 & 0.30 \\
$\mathbf{G e}_{22} \mathbf{S b}_{22} \mathbf{T e}_{56}$ & $r_{i j}^{m}(\AA)$ & 3.05 & 3.34 & 3.20 & 3.51 & 3.50 & 3.28 \\
& & 0.74 & 3.08 & 0.59 & 1.01 & 2.83 & 0.59 \\
\hline \hline
\end{tabular}

$\mathrm{Ge}_{10} \mathrm{Sb}_{10} \mathrm{Te}_{80}$ liquid. We finally note that the homopolar Ge-Ge [Fig. 10(c)] and Sb-Sb [Fig. 10(d)] do not give rise to the same bond angle distribution because the former leads to a broad distribution centered at $90^{\circ}$, and extending up to $180^{\circ}$, whereas the latter has a much more sharp distribution and $\mathrm{Sb}$-Sb-Te angles larger than $\simeq 140^{\circ}$ are virtually absent.

Taking into account the dispersion forces leads to a shift of the Te-Ge-Te bond angle distribution to lower angles [broken curve in Fig. 9(c)], whereas virtually no changes are seen in the corresponding Te-Sb-Te distribution. This indicates that Eq. (1) leads to an increased number of octahedral geometries, as also revealed from the increased contribution at $\simeq 160^{\circ}$, a change that is only moderately acknowledge by a $\mathrm{Ge}$ coordination number increase (Table III).

TABLE III. Calculated coordination numbers $n_{i}$ at different compositions $x$ in the $(820 \pm 20 \mathrm{~K}) \mathrm{Ge}_{x} \mathrm{Sb}_{x} \mathrm{Te}_{100-2 x}$ liquid at the cutoff distance $r_{i j}^{m}$ determined from the minimum of corresponding functions $g_{i}(r)$. Calculations for $\mathrm{Ge}_{14} \mathrm{Sb}_{14} \mathrm{Te}_{72}$ liquid are performed in presence or absence of the dispersion correction [Eq. (1)]. The coordination numbers of $\mathrm{Ge}_{22} \mathrm{Sb}_{22} \mathrm{Te}_{56}$ are also calculated (bottom of the table) at a fixed cutoff distance of $3.2 \AA$, in order to be comparable with those of a liquid at $900 \mathrm{~K}$ [9].

\begin{tabular}{lcccc}
\hline \hline System & & $n_{\mathrm{Ge}}$ & $n_{\mathrm{Sb}}$ & $n_{\mathrm{Te}}$ \\
\hline $\mathbf{G e}_{6} \mathbf{S b}_{6} \mathbf{T e}_{88}$ & $r_{i}^{m}(\AA)$ & 3.20 & 3.58 & 3.48 \\
& & $4.13 \pm 0.05$ & $4.93 \pm 0.10$ & $3.71 \pm 0.09$ \\
$\mathbf{G e}_{10} \mathbf{S b}_{10} \mathbf{T e}_{80}$ & $r_{i}^{m}(\AA)$ & 3.30 & 3.63 & 3.54 \\
& & $4.33 \pm 0.05$ & $5.05 \pm 0.09$ & $3.91 \pm 0.09$ \\
$\mathbf{G e}_{14} \mathbf{S b}_{14} \mathbf{T e}_{72}$ & $r_{i}^{m}(\AA)$ & 3.24 & 3.68 & 3.52 \\
& & $4.29 \pm 0.05$ & $5.48 \pm 0.10$ & $3.93 \pm 0.09$ \\
$\mathbf{G e}_{14} \mathbf{S b}_{14} \mathbf{T e}_{72}$ & $r_{i}^{m}(\AA)$ & 3.19 & 3.70 & 3.52 \\
without Eq. $(1)$ & $4.38 \pm 0.05$ & $5.86 \pm 0.09$ & $4.27 \pm 0.10$ \\
$\mathbf{G e}_{22} \mathbf{S b}_{22} \mathbf{T e}_{56}$ & $r_{i}^{m}(\AA)$ & 3.27 & 3.51 & 3.48 \\
& & $4.40 \pm 0.05$ & $4.56 \pm 0.09$ & $3.61 \pm 0.09$ \\
$\mathbf{G e}_{22} \mathbf{S b}_{22} \mathbf{T e}_{56}$ & $r_{i}^{m}(\AA)$ & 3.20 & 3.20 & 3.20 \\
$820 \mathrm{~K}$ & & $4.20 \pm 0.05$ & $3.15 \pm 0.10$ & $2.48 \pm 0.07$ \\
$900 \mathrm{~K}, \mathbf{R e f}_{\text {. }}$ (9] & & 4.3 & 3.4 & 2.8 \\
\hline \hline
\end{tabular}



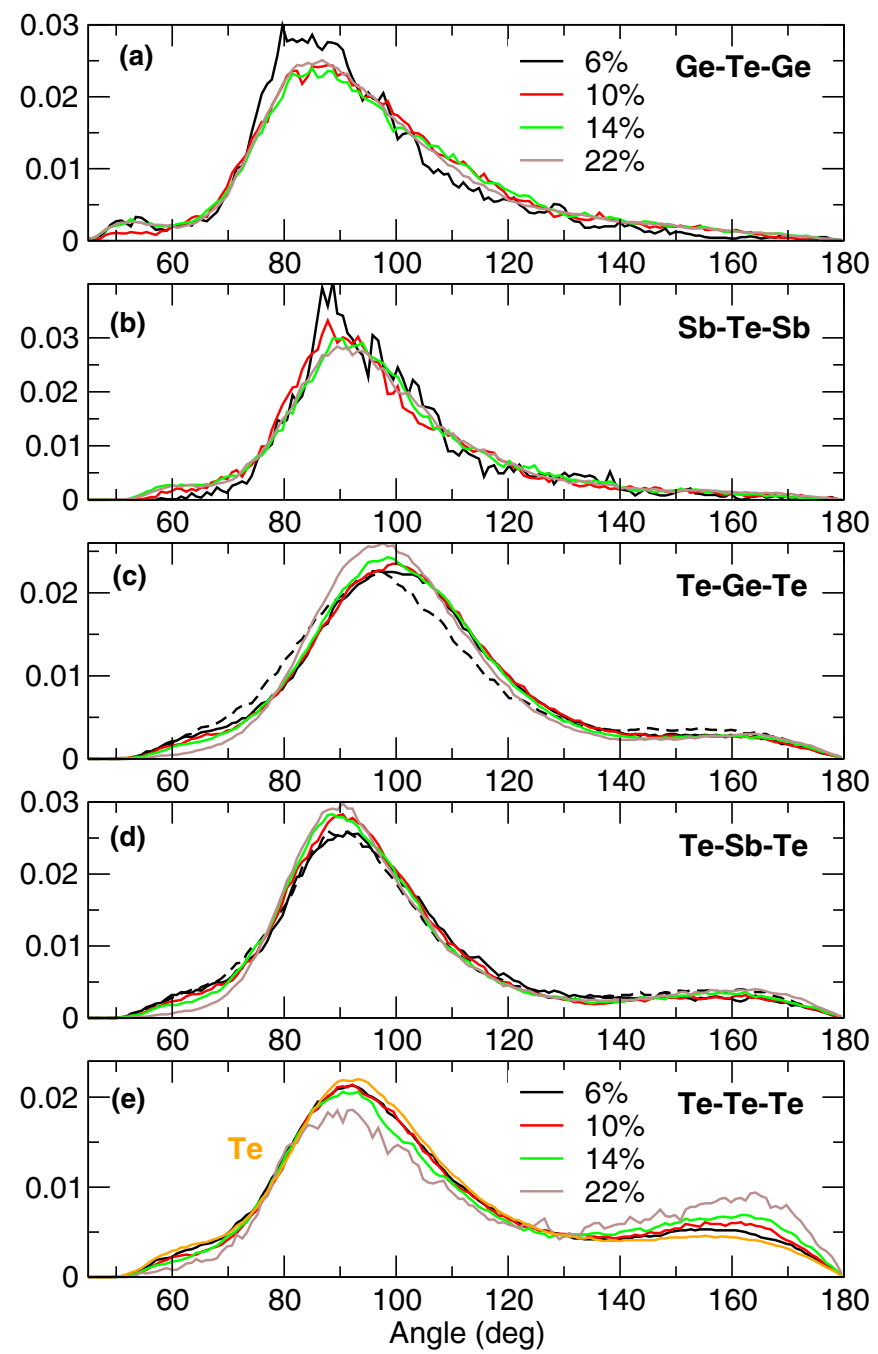

FIG. 9. (Color online) Evolution of the simulated bond angle distributions Ge-Te-Ge (a), Sb-Te-Sb (b), Te-Ge-Te (c), Te-Sb-Te (d), and Te-Te-Te (e) at fixed temperature $(820 \pm 20 \mathrm{~K})$ as a function of composition $x$ in $\mathrm{Ge}_{x} \mathrm{Sb}_{x} \mathrm{Te}_{100-2 x}$ liquids. In (e), the orange curve represents the Te-Te-Te bond angle distribution of liquid Te. In (c) and (d), the broken black curve represents results of liquid $\mathrm{Ge}_{14} \mathrm{Sb}_{14} \mathrm{Te}_{72}$ from a simulation without the Grimme correction.

\section{F. Ring statistics}

We also characterize intermediate range order that is determined from the ring statistics which has been found to be especially relevant for the understanding of the amorphous to crystal transition. Indeed, Akola and Jones [8,9] have emphasized the fact that so-called $\mathrm{ABAB}$ squares (a fourmembered ring, Fig. 11) play a crucial role in this transition occuring during the phase change mechanism. Such ABAB rings $(A=G e, S b$ and $B=T e)$ refer to even-membered rings with perfect bond alternation, each $\mathrm{Ge} / \mathrm{Sb}$ is connected to a Te atom inside the loop, and these fully heteropolar rings represent the very basic crystalline motif encountered in the crystalline structure of GST225.

We have calculated the ring statistics as a function of Te concentration by using an algorithm that is mostly based on the King [47]-Franzblau [48] shortest path search, and which is
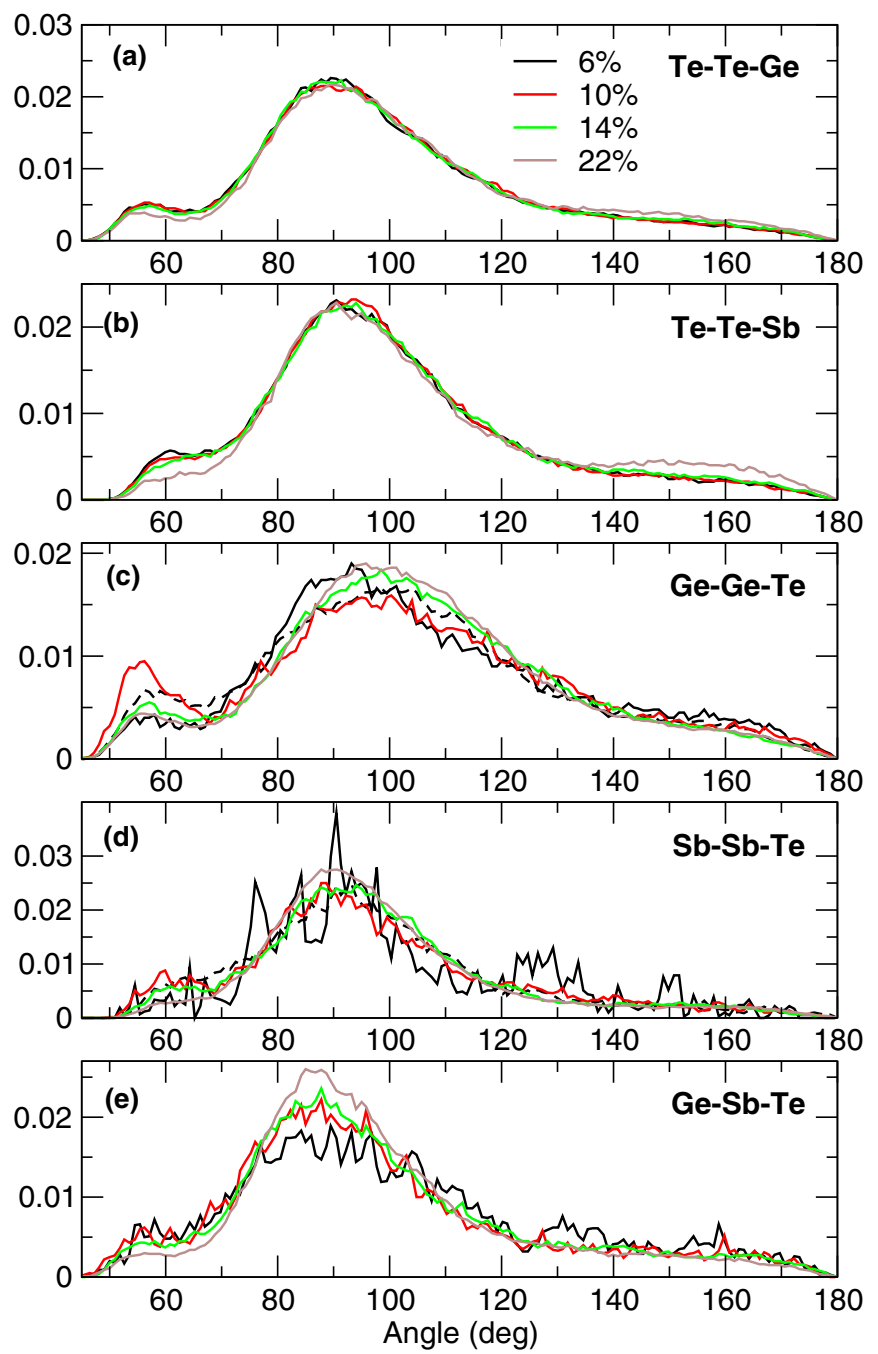

FIG. 10. (Color online) Evolution of the simulated bond angle distributions Te-Te-Ge (a), Te-Te-Sb (b), Ge-Ge-Te (c), Sb-Sb-Te (d), and Ge-Sb-Te (d) at fixed temperature $(820 \pm 20 \mathrm{~K})$ as a function of composition $x$ in $\mathrm{Ge}_{x} \mathrm{Sb}_{x} \mathrm{Te}_{100-2 x}$ liquids. In (c) and (d), the broken black curve represents results of liquid $\mathrm{Ge}_{14} \mathrm{Sb}_{14} \mathrm{Te}_{72}$ from a simulation without the Grimme correction.

now part of the Rigorous Investigation of networks generated using simulation (RINGS) code [49]. The statistics is computed up to rings of size $n=10$, and a cutoff distance of $3.3 \AA$ has been used for all atomic pairs. Minor changes are found under a cut-off change of $\pm 0.1 \AA$ (see also Fig. 13).

Figure 12 shows the ring statistics $\mathrm{R}^{(n)}$ for the five compositions of liquid $\mathrm{Ge}_{x} \mathrm{Sb}_{x} \mathrm{Te}_{100-2 x}$. First, one should note that, in contrast with oxide glasses $\left(\mathrm{SiO}_{2}, \mathrm{GeO}_{2}\right)$ having $100 \%$ heteropolar bonds and only even sized rings [50,51], chalcogenides can have both even and odd sized rings because of the presence of homopolar bonds [23,52-54], and this turns out to be also the case for the present telluride system.

\section{Odd and even rings}

For elemental $\mathrm{Te}$, one nearly recovers results from a previous analysis of Akola and co-workers [32], i.e., the ring distribution is dominated by $n=4$ and 7 membered 


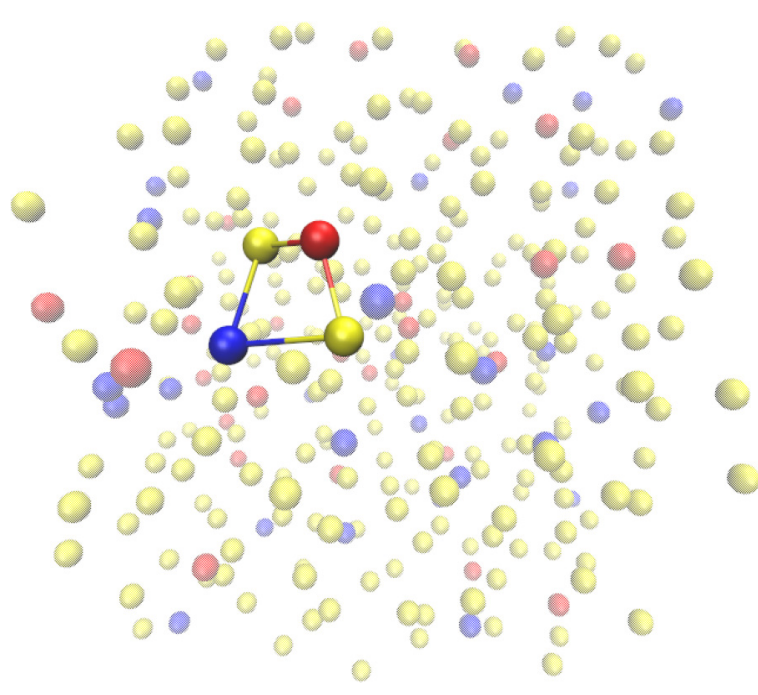

FIG. 11. (Color online) A typical ABAB ring found in liquid $\mathrm{Ge}_{14} \mathrm{Sb}_{14} \mathrm{Te}_{72}$. Ge, $\mathrm{Sb}$, and $\mathrm{Te}$ atoms are in red, blue, and yellow, respectively.

rings which can, in certain situations, be made out of two neighboring Te chains. Addition of the $\mathrm{Ge} / \mathrm{Sb}$ atoms leads to a growth of larger rings $n=8-10,6$, and 5 rings, the latter becoming the dominant ring structure for undercooled liquid GST225 given that about 55 of such rings are detected in the structure (and $R^{(4)}=44$ ). Some triangular paths are also

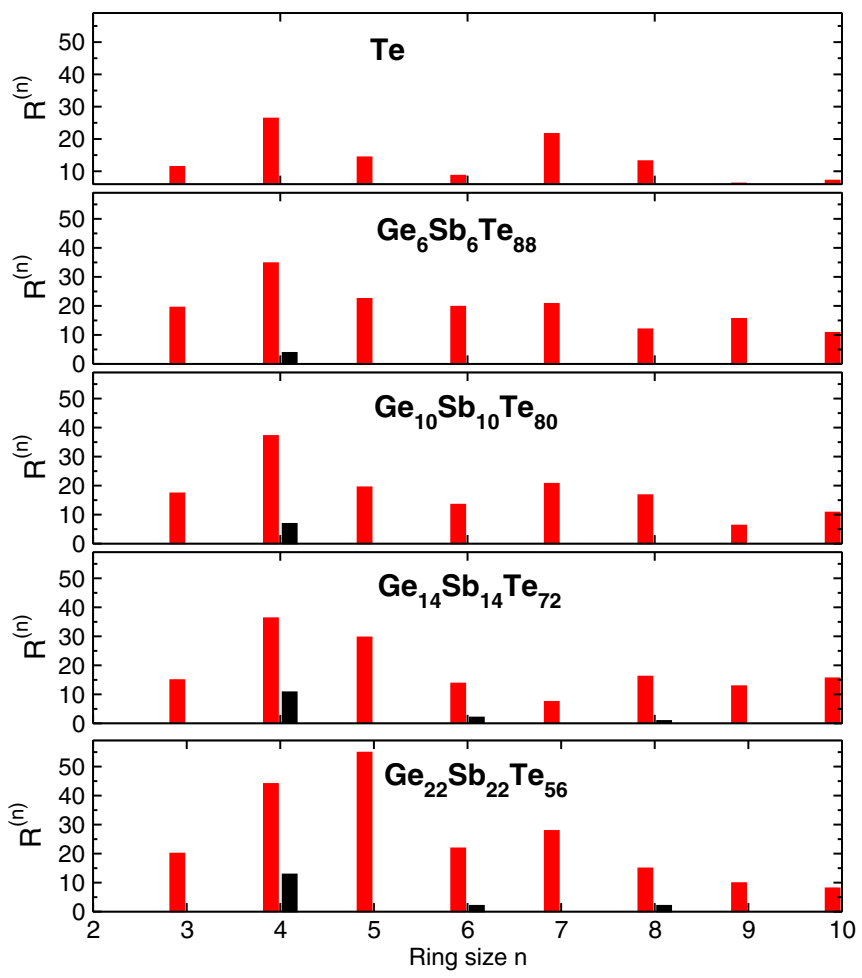

FIG. 12. (Color online) Calculated number of rings $R^{(n)}$ in liquids $\mathrm{Ge}_{x} \mathrm{Sb}_{x} \mathrm{Te}_{100-2 x}$ for different compositions at $820 \mathrm{~K}$ using the RINGS method [49]. The red and black bars correspond to a full ring analysis (odd and even sized rings) and to the $\mathrm{ABAB}$ analysis, respectively. Here, $\mathrm{ABAB}$ refers to even rings with a perfect (heterpolar) bond alternation.

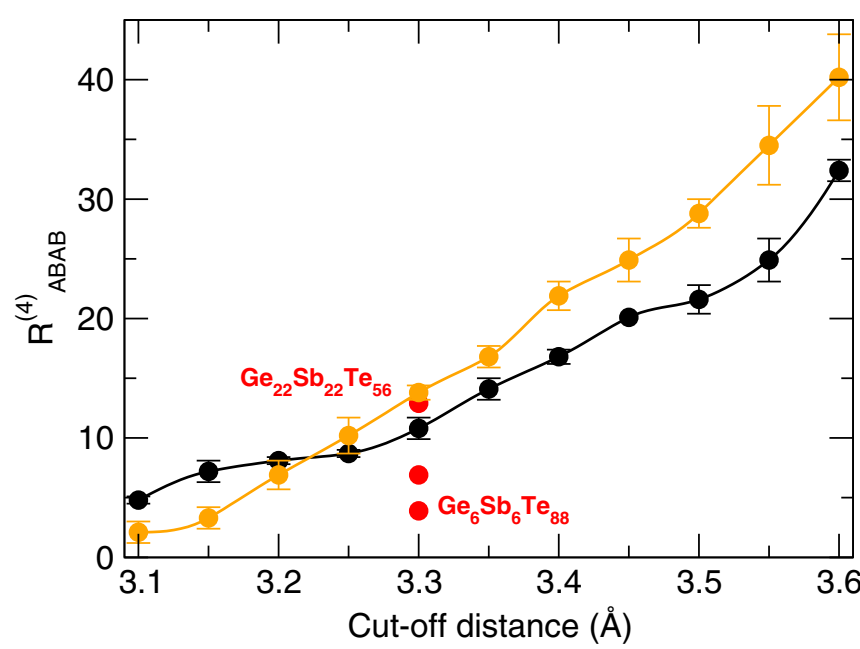

FIG. 13. (Color online) Calculated number of ABAB rings $R^{(4)}$ in liquid $(820 \mathrm{~K}) \mathrm{Ge}_{14} \mathrm{Sb}_{14} \mathrm{Te}_{72}$ as a function of the cutoff in two situations: presence (black) or absence (orange) of the dispersion correction [Eq. (1)]. Red circles indicate the ABAB fraction for the other Ge-Sb-Te compositions (same as Fig. 12).

found but they represent a minority $\left(R^{(3)} \simeq 10\right.$ for nearly all compositions).

\section{Emergence of $A B A B$ motifs}

Of special interest is the behavior of the $\mathrm{ABAB}$ motifs, which are found to dominate the $\mathrm{ABAB}$ statistics given that only very weak contributions are found for $n=6$ and 8 for all compositions, similarly to an analysis [9] performed on liquid (900 K) GST225.

ABAB motifs grow continuously with increasing $\mathrm{Ge} / \mathrm{Sb}$ content. They are barely present for the $6 \%$ composition, and for this liquid the 4-membered rings are mostly made of homopolar Te-Te bonds (89\%). This ratio tends to decrease as more and more $\mathrm{Ge} / \mathrm{Sb}$ atoms are inserted into the network structure. In the GST225 liquid (22\%), we find that ABAB motifs represents about $27 \%$ of the four-membered ring distribution (i.e., $13 \mathrm{ABAB}$ rings over a total population of 44 4-rings), this fraction (0.036 $\mathrm{ABAB}$ ring/atom) being smaller than the one found (0.080 ABAB ring/atom) for the similar liquid [9], which is been simulated at a different temperature $(900 \mathrm{~K})$, and using a slightly different modeling scheme. In order to investigate the effect of the Grimme correction and the consequence of a reduction of the bond distances, we represent in Fig. 13 the population $\mathrm{R}^{(4)}$ of $\mathrm{ABAB}$ motifs of the liquid $\mathrm{Ge}_{14} \mathrm{Sb}_{14} \mathrm{Te}_{72}$ as a function of the cutoff. The plot reveals that the presence of dispersion forces will reduce the fraction of $\mathrm{ABAB}$ rings whatever the chosen cutoff $r>r_{\min }$.

\section{VIBRATIONNAL AND ELECTRONIC PROPERTIES}

\section{A. Vibrational density of states}

The experimental vibrational density of states (VDOS) $g(\omega)$ of the $\mathrm{Ge}_{x} \mathrm{Sb}_{x} \mathrm{Te}_{100-2 x}$ liquids are presented in Fig. 14. For all composition, VDOS are characterized by two vibrational modes localized around 5 and $20 \mathrm{meV}$ and it is difficult from 


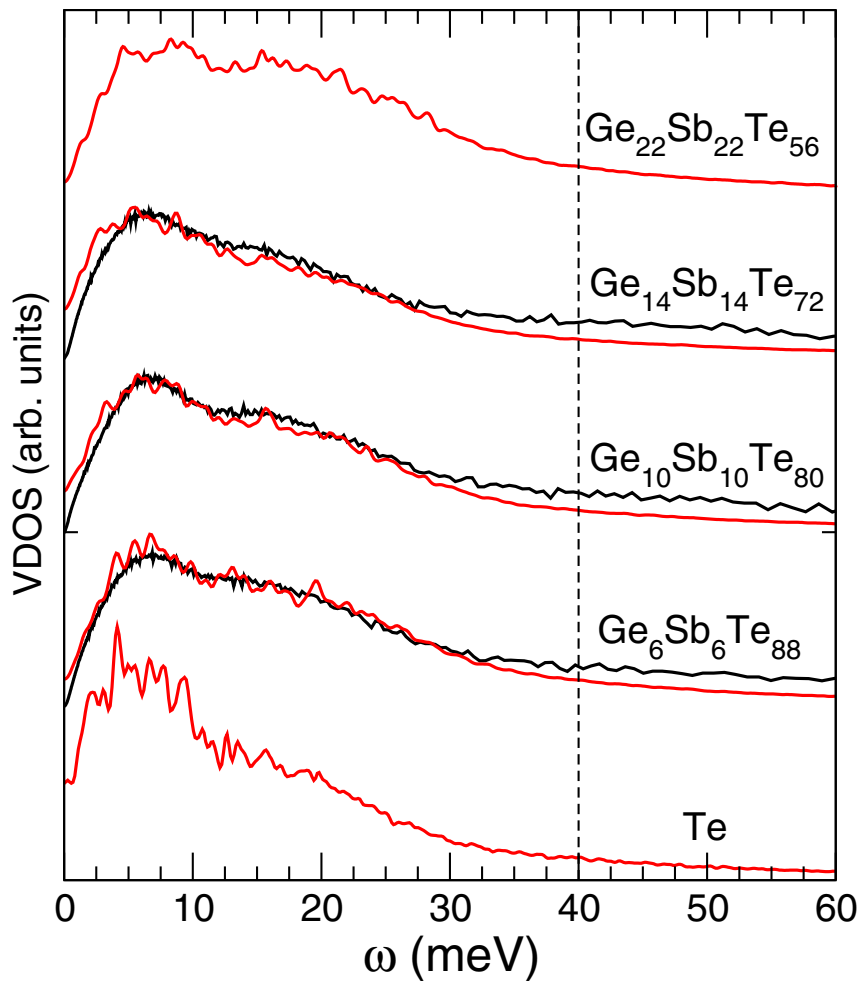

FIG. 14. (Color online) Direct comparison between the experimental vibrational density of states (black lines), and the calculated ones (red lines) at $T \simeq 820 \mathrm{~K}$ as a function of Te concentration in Ge$\mathrm{Sb}$-Te liquids. The vertical broken line signals the approximate limit beyond which the agreement between simulated and experimental VDOS is decreasing markedly.

a simple inspection to evidence changes as the composition varies. In order to quantify the variations, experimental VDOS were fitted between 0.9 and $40 \mathrm{meV}$ using the sum of two Lorentzian functions as proposed in Ref. [5]. As seen from the center values listed in Table IV, the low-energy part of the spectra remains stable in composition, while the center of the second Lorentzian shift toward high energy when the $\mathrm{Ge} / \mathrm{Sb}$ content increases.

The calculated vibrational density of states $g(\omega)$ of the different Ge-Sb-Te liquids are compared in Fig. 14 to the experimental measurements, using the Fourier transform of the velocity-velocity autocorrelation function:

$$
g(\omega)=\frac{1}{N k_{B} T} \sum_{j=1}^{N} \int_{-\infty}^{\infty}\left\langle\mathbf{v}_{j}(t) \mathbf{v}_{j}(0)\right\rangle e^{i \omega t} d t .
$$

As seen from Fig. 14, a large part of the spectra is very well reproduced, this statement being valid for all considered compositions. Similarly to our study of other liquid tellurides [5,26], we find that the calculated VDOS deviates from the experimental spectra for $\omega \geqslant 30 \mathrm{meV}$ because a more important decrease to zero is obtained at high frequency. It should be reminded, however, that since the experimental available $E=\hbar \omega$ range is correlated with a finite $k$ range, the measured $g(\omega)$ is slightly distorted and incomplete.

We note that for elemental tellurium, the VDOS consists in an important contribution at low frequency $(\omega<10 \mathrm{meV})$
TABLE IV. Centers of two Lorentzian functions (in meV) used to fit the experimental VDOS.

\begin{tabular}{lccc}
\hline \hline & $\mathbf{G e}_{6} \mathbf{S} \mathbf{b}_{6} \mathbf{T} \mathbf{e}_{88}$ & $\mathbf{G e}_{10} \mathbf{S} \mathbf{b}_{10} \mathbf{T} \mathbf{e}_{80}$ & $\mathbf{G e}_{14} \mathbf{S} \mathbf{b}_{14} \mathbf{T} \mathbf{e}_{72}$ \\
\hline$T(\mathrm{~K})$ & 843 & 823 & 823 \\
Peak 1 & $6.1(1)$ & $6.2(1)$ & $6.2(1)$ \\
Peak 2 & $15.4(5)$ & $16.3(4)$ & $16.8(4)$ \\
\hline \hline
\end{tabular}

signaling also the possibility of low frequency (floppy) modes $(5 \mathrm{meV})$ that are typical of flexible glass-forming liquids [55]. As the Te content is reduced, this low frequency band tends to reduce in intensity and is driven by the progressive stiffening of the liquid structure due to the addition of $\mathrm{Ge} / \mathrm{Sb}$ cross-links. Simultaneously, a second broad band centered around $20 \mathrm{meV}$ builds up with the concentration $x$.

When the partial contribution to the VDOS are considered (Fig. 15), one realizes, indeed, that the dominant contribution at low frequency arises, indeed, mostly from Te atoms, this statement being valid even at smaller Te content. This is also consistent with the fact the chalcogens contribute to
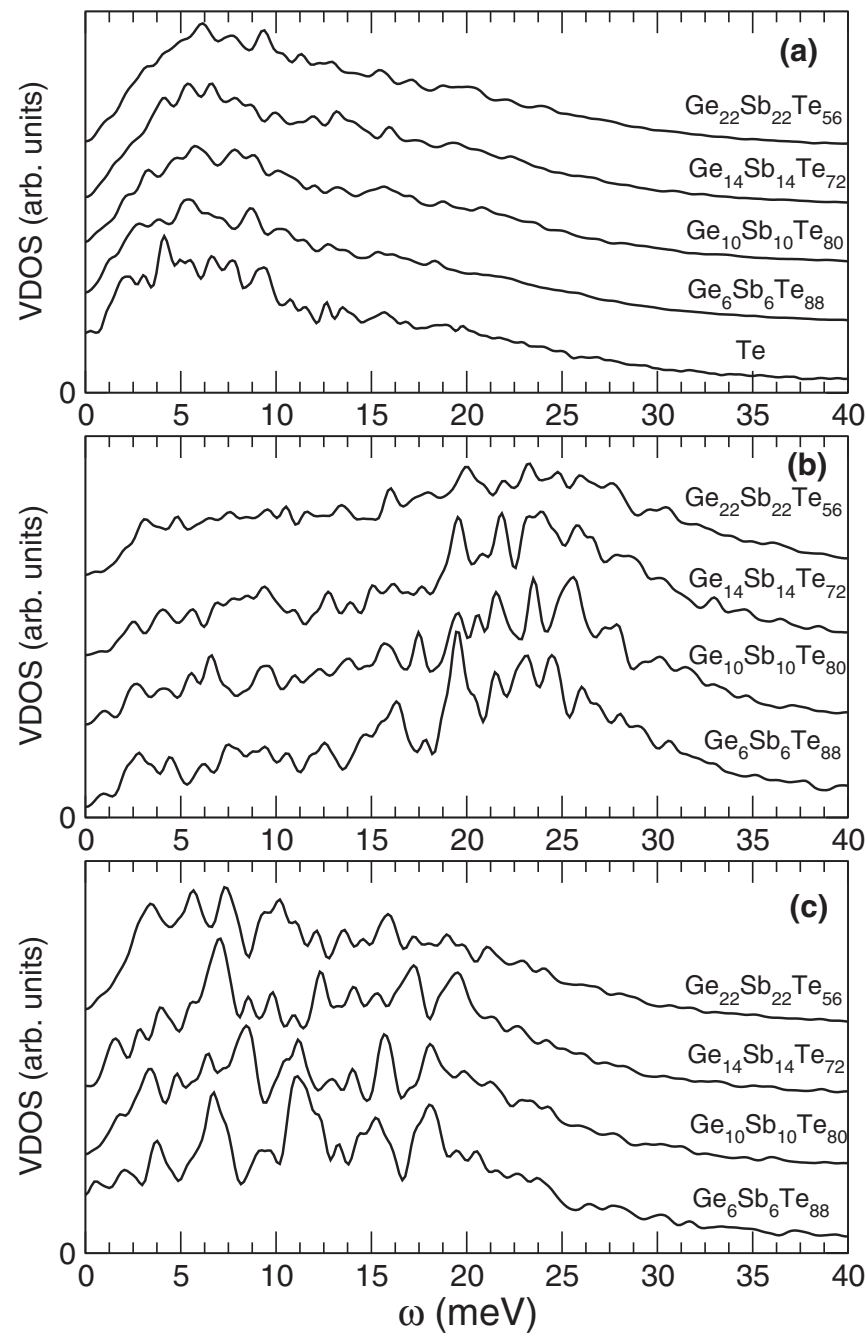

FIG. 15. Calculated partial vibrational density of states (VDOS) for different Ge-Sb-Te liquids at fixed temperature $(820 \mathrm{~K})$. Contributions from $\mathrm{Te}(\mathrm{a}), \mathrm{Ge}(\mathrm{b})$, and $\mathrm{Sb}$ (c) atoms. 


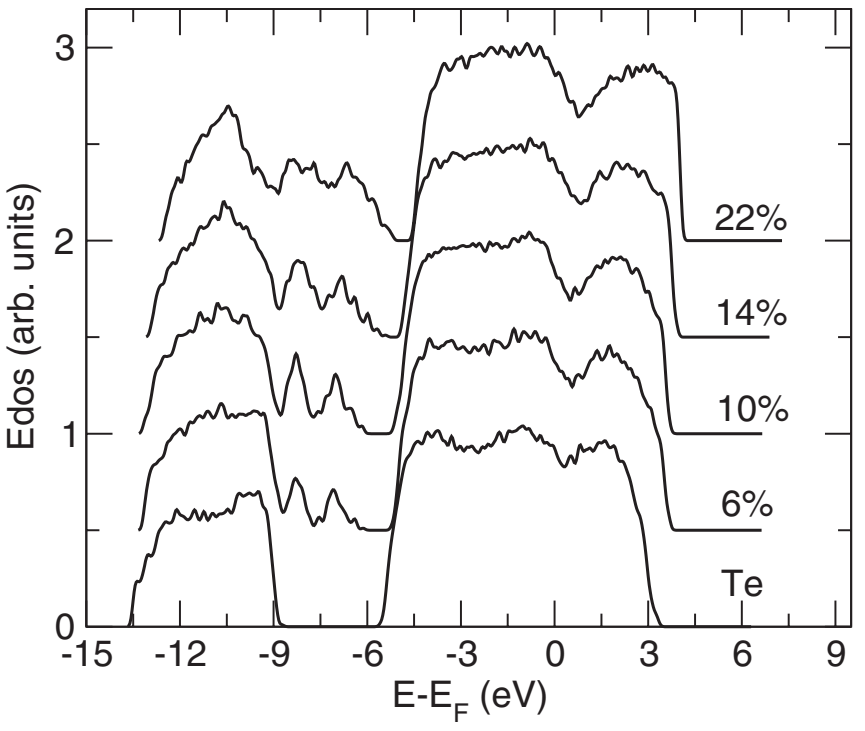

FIG. 16. Calculated electronic density of states (EDOS) for different Ge-Sb-Te liquids at fixed temperature $(820 \pm 20 \mathrm{~K})$.

flexibility and low-frequency modes [22]. Finally, Ge atoms mostly contribute to the broad band at $20-25 \mathrm{meV}$ [Fig. 15(b)], whereas $\mathrm{Sb}$ atoms have a dominant contribution at $5-20 \mathrm{meV}$ [Fig. 15(c)].

\section{B. Electronic density of states}

Finally, we represent in Fig. 16 the calculated electronic density of states which exhibits a three-band structure located at $-10,-7$, and $-3 \mathrm{eV}$. The lowest contribution around $\simeq-10 \mathrm{eV}$ is associated with s electrons, whereas the broad band between $-5 \mathrm{eV}$ and the Fermi energy has related to $\mathrm{p}$ electrons. The intermediate energies found between -9 and $-6 \mathrm{eV}$ are related to antibonding $\sigma$ electronic states, and related to $\mathrm{Ge}$ and $\mathrm{Sb}$ atoms as discussed next. When compared to available x-ray photoemission spectroscopy (XPS) [56] obtained for the GST225 compound in the amorphous state, it is found that the main band structure is reproduced but with bands that are found to be broader when compared to a-GST225, and a gap at the Fermi energy that is closed and which signals the metallicity of the liquid.

When the EDOS is studied as a function of Te content, several important features emerge, indeed. First, the gap between the $\sigma$ band found at energies lower than $-9 \mathrm{eV}$, and the $\pi$ bonding energies close to the Fermi energy, tends to disappear as the fraction of $\mathrm{Ge} / \mathrm{Sb}$ is increased. In elemental $\mathrm{Te}$, it leads to a wide gap given the electronic states of $\mathrm{Te}$ atoms made of $\sigma$ bonds involving the $4 s$ orbitals, and the $p$ and $d$ electronic states at higher energies, which populate the valence band, and contribute to the interchain interaction [32]. However, this gap is still observable at the lower edge of the $p$ band $(\simeq-6 \mathrm{eV})$ for the highest concentration $x$ (GST225, $x=22 \%$ ), a behavior that has been already noticed for the same system in the amorphous and liquid state [9], and which results from a small $s-p$ mixing for all investigated compositions.
For the intermediate compositions (6\%-14\%), it is seen that contributions at -7 and $-8 \mathrm{eV}$ increase, and an atomic projection allows to associate these contributions with the $s$ electrons of germanium and antimony, respectively. The $\mathrm{Ge}$ and $\mathrm{Sb} p$ orbitals are also present in the conduction band, and these are responsible for an increased pseudogap at the Fermi energy with $\mathrm{Ge} / \mathrm{Sb}$ content, leading to a more pronounced separation of the valence and conduction bands. In elemental $\mathrm{Te}$, this gap is absent or, at least, barely visible, as also reported in Ref. [32].

\section{CONCLUSION}

Chalcogenides using $\mathrm{Ge}, \mathrm{Sb}$, and $\mathrm{Te}$ represent a very important class of materials with tremendous potentialities in terms of optoelectronic applications. They also represent promising base material for applications with enhanced possibilities when properly alloyed with additives. This leads to specific properties that are continuously optimized with chemical composition. Still, the way these additives affect the properties is strongly influenced by the underlying Ge-Sb-Te network structure, and its behavior with Te content. The description of structural properties of such tellurides appears, thus, to be a fundamental issue in optoelectronics with, obviously, a high degree of possible applications that could emerge from an increased understanding.

Here, we have focused on the structural, vibrational and electronic properties of three compositions in the liquid Ge-SbTe binary by combining molecular dynamics simulations, neutron diffraction and inelastic neutron scattering experiments. For completeness, we have also simulated two additional compositions (Te and GST225). In the ternary Ge-Sb-Te phase diagram, experimental and theoretical focus is usually made on the pseudo-tie-line $\mathrm{GeTe}-\mathrm{Sb}_{2} \mathrm{Te}_{3}$, i.e., along a compositional join which contains a nearly fixed amount of Te atoms. We have adopted a different strategy by investigating the properties along the $\mathrm{Ge}_{x} \mathrm{Sb}_{x} \mathrm{Te}_{100-2 x}$ join, a strategy that was guided by previous findings [15] indicating that a flexible to rigid transition may be expected close to the $\mathrm{GeTe}_{4}-\mathrm{SbTe}_{4}$ join.

The study of the three main compositions $\mathrm{Ge}_{6} \mathrm{Sb}_{6} \mathrm{Te}_{88}$, $\mathrm{Ge}_{10} \mathrm{Sb}_{10} \mathrm{Te}_{80}$, and $\mathrm{Ge}_{14} \mathrm{Sb}_{14} \mathrm{Te}_{72}$ reveals that both the experimental pair correlation function in real space, and the structure factor in reciprocal space can be reproduced from molecular simulations with an accuracy of unprecedented quality. This central result is obtained by solving the wellknown bond distance problem (an overestimation of bond distances) encountered in the modeling of typical liquid and amorphous tellurides. We arrive to such an agreement by incorporating dispersion forces in the DFT simulation, which are handled in a semi-empirical way by a Grimme correction. An immediate first result is that the typical bond distances of the Ge-Sb-Te liquids are not only reproduced with confidence, but also substantially reduced when such forces are present. Having obtained a good reproduction of the first peak and first minimum of the function $g(r)$, a second immediate consequence of the simulations is that obtained coordination numbers are decreased with respect to previous findings, especially when they are directly compared to simulations without the Grimme correction for a selected composition $\left(\mathrm{Ge}_{14} \mathrm{Sb}_{14} \mathrm{Te}_{72}\right)$. Finally, it has been found that the presence of 
$A B A B$ rings which are proposed to be the relevant motifs for the amorphous to crystal transition in phase change materials, are also largely dependent on the simulation scheme, and dispersion forces tend to reduce the number of such rings in the $\mathrm{Ge}_{14} \mathrm{Sb}_{14} \mathrm{Te}_{72}$ liquid, albeit unable to rule out this obvious structural motif which is able to explain the enhanced crystallization tendency of such materials.

For all compositions, the structure of these Ge-Sb-Te liquids is made of a majority of germanium and antimony atoms in a defective octahedral geometry, a result that is determined from both the coordination numbers and the bond angle distributions. The analysis of the vibrational properties shows an excellent agreement between simulated and experimental data, this statement being true for all investigated compositions.

This study also reveals that important structural changes take place between the $14 \%$ composition and the standard GST225 phase change undercooled liquid that manifests by notable differences in the partial $\mathrm{Ge}-\mathrm{Te}$ and $\mathrm{Sb}$-Te partial structure factors, and in partial pair correlation functions. One, finally, acknowledges that homopolar bonds (Ge-Ge and $\mathrm{Sb}-\mathrm{Sb})$ start to increase in a visible fashion for $x>10 \%$, i.e., close to the predicted flexible to rigid threshold [15], and in line with similar MD studies on other chalcogenides (Ge-Te [23] and As-Se [57]). It would be interesting to probe if such behavior are also seen at lower temperatures and in the amorphous phase. Work in this direction is in progress.

\section{ACKNOWLEDGMENTS}

The authors thank P. Boolchand, K. Gunasekera, and A. Kachmar for useful and stimulating discussions. Support from Agence Nationale de la Recherche (ANR) (Grant No. ANR11-BS08-0012) is gratefully acknowledged. GENCI (Grand Equipement National de Calcul Intensif) is acknowledged for supercomputing access.
[1] Phase Change Materials and Applications, edited by S. Raoux, and M. Wuttig (Springer, Berlin, 2008).

[2] S. Danto et al., Adv. Funct. Mater. 16, 1847 (2006).

[3] C. Steimer, M. V. Coulet, W. Welnic, H. Dieker, R. Detemple, C. Bichara, B. Beuneu, J. P. Gaspard, and M. Wuttig, Adv. Mater. 20, 4535 (2008).

[4] A. V. Kolobov, P. Fons, M. Krbal, R. E. Simpson, S. Hosokawa, T. Uruga, H. Tanida, and J. Tominaga, Appl. Phys. Lett. 95, 241902 (2009)

[5] C. Otjacques, J.-Y. Raty, M.-V. Coulet, M. Johnson, H. Schober, C. Bichara, and J.-P. Gaspard, Phys. Rev. Lett. 103, 245901 (2009).

[6] M. Delheusy, J. Y. Raty, R. Detemple, W. Welnic, M. Wuttig, and J. P. Gaspard, Physica B 350, E1055 (2004).

[7] C. Bichara, M. Johnson, and J.-P. Gaspard, Phys. Rev. B 75, 060201 (2007).

[8] J. Akola and R. O. Jones, Phys. Rev. B 76, 235201 (2007).

[9] J. Akola and R. O. Jones, J. Phys. Cond. Matt. 20, 465103 (2008).

[10] J. Kalikka, J. Akola, J. Larrucea, and R. O. Jones, Phys. Rev. B 86, 144113 (2012).

[11] T. H. Lee and S. R. Elliott, Phys. Rev. Lett. 107, 145702 (2011).

[12] J. Akola and R. O. Jones, Phys. Status Solidi 249, 1851 (2012).

[13] F.-C. Pang, D. Wang, N.-K. Chen, S.-Y. Xie, X. Meng, C.-S. Huo, H. Yang, X.-P. Su, W.-Q. Wang, and H.-L. Tu, Comp. Mater. Sci. 61, 287 (2012).

[14] P. Lebaudy, J. M. Saiter, J. Grenet, M. Belhadji, and C. Vautier, Mater. Sci. Eng., A 132, 273 (1991).

[15] M. Micoulaut, J.-Y. Raty, C. Otjacques, and C. Bichara, Phys. Rev. B 81, 174206 (2010).

[16] I. Kaban, Th. Halm, W. Hoyer, P. Jòvari, and J. Neuefeind, J. Non-Cryst. Solids 326-327, 120 (2003).

[17] S. Sen, T. G. Edwards, J.-Y. Cho, and Y.-C. Joo, Phys. Rev. Lett. 108, 195506 (2012).

[18] M. Micoulaut, K. Gunasekera, S. Ravindren, and P. Boolchand, Phys. Rev. B 90, 094207 (2014).

[19] J. Kalikka, J. Akola, R. O. Jones, S. Kohara, and T. Usuki, J. Phys. Cond. Matt. 24, 015802 (2012).
[20] E. Cho, J. Im, C. Park, W. J. Son, D. H. Kim, H. Horii, J. Ihm, and S. Han, J. Phys. Cond. Matt. 22, 205504 (2010).

[21] M. Micoulaut, A. Kachmar, Th. Charpentier, Phys. Status Solidi B 249, 1890 (2012).

[22] Rigidity Theory and Applications, edited by M. F. Thorpe and P. M. Duxburry (Plenum Press, New York, 1999).

[23] A. A. Piarristeguy, M. Micoulaut, R. Escalier, P. Jóvári, I. Kaban, J. Van Eijk, J. Luckas, S. Ravindren, P. Boolchand, and A. Pradel, J. Chem. Phys. 143, 074502 (2015).

[24] J. Frayret, E. Barthélémy, S. Albert, C. Vigreux, and A. Pradel, Optoelectr. Adv Mater. 3, 260 (2009).

[25] M. Micoulaut, J. Chem. Phys. 138, 061103 (2013).

[26] M. Micoulaut, M.-V. Coulet, A. Piarristeguy, M. R. Johnson, G. J. Cuello, C. Bichara, J.-Y. Raty, H. Flores-Ruiz, and A. Pradel, Phys. Rev. B 89, 174205 (2014)

[27] H. E. Fischer, G. J. Cuello, P. Palleau, D. Feltin, A. C. Barnes, Y. S. Badyal, and J. M. Simonson, Appl. Phys. A 74, S160 (2002).

[28] CORRECT: A correction program for neutron diffraction data, Version 2.4, M. A. Howe, R. L. McGreevy, and P. Zetterstrm (1998).

[29] LAMP, the Large Array Manipulation Program. http://www.ill.fr/ data_treat/lamp/the_lamp-book.

[30] B. Legendre, C. Hancheng, S. Bordas, and M. T. ClavagueraMora, Thermochimica Acta 78, 141 (1984)

[31] Y. Tsuchiya, J. Phys. Soc. Jpn. 60, 227 (1991).

[32] J. Akola, R. O. Jones, S. Kohara, T. Usuki, and E. Bychkov, Phys. Rev. B 81, 094202 (2010).

[33] R. Car and M. Parrinello, Phys. Rev. Lett. 55, 2471 (1985).

[34] C. Bergman, C. Bichara, J.-P. Gaspard, and Y. Tsuchiya, Phys. Rev. B 67, 104202 (2003).

[35] N. Troullier and J. L. Martins, Phys. Rev. B 43, 1993 (1991).

[36] S. Nosé, Mol. Phys. 52, 255 (1984); W. G. Hoover, Phys. Rev. A 31, 1695 (1985)

[37] S. Grimme, J. Comput. Chem. 27, 1787 (2006).

[38] A. Zeidler et al., Phys. Rev. Lett. 113, 135501 (2014).

[39] M. Micoulaut, R. Vuilleumier, and C. Massobrio, Phys. Rev. B 79, 214205 (2009). 
[40] Landolt-Börnstein New Series I/16A Ed Schopper (Berlin, Springer, 2000).

[41] We relate the position $r$ of a peak in real space to the position $k$ of a corresponding peak in Fourier space by using the relation $k r \approx 7.7$, which identifies the location of the first maximum of the spherical Bessel function $j_{0}(k r)$.

[42] L. Verlet, Phys. Rev. 165, 201 (1968).

[43] J. P. Hansen and D. Schiff, Mol. Phys. 25, 1281 (1973).

[44] R. Evans and T. J. Sluckin, J. Phys. C 14, 2569 (1981).

[45] J. D. Weeks, D. Chandler, and H. C. Andersen, J. Chem. Phys. 54, 5237 (1971).

[46] L. Pauling, Nature of the Chemical Bond (Cornell University Press, Ithaca, NY, 1960).

[47] V. King, Nature (London) 213, 1112 (1967).

[48] D. S. Franzblau, Phys. Rev. B 44, 4925 (1991).

[49] S. Le Roux and P. Jund, Comput. Mater. Sci. 49, 70 (2010); 50, 1217 (2011)
[50] X. Yuan and A. N. Cormack, Comp. Mater. Sci. 24, 343 (2002).

[51] M. Micoulaut, X. Yuan, and L. W. Hobbs, J. Non-Cryst. Solids 353, 1951 (2007).

[52] M. Micoulaut, A. Kachmar, M. Bauchy, S. Le Roux, C. Massobrio, and M. Boero, Phys. Rev. B 88, 054203 (2013)

[53] S. L. Roux, A. Zeidler, P. S. Salmon, M. Boero, M. Micoulaut, and C. Massobrio, Phys. Rev. B 84, 134203 (2011).

[54] M. Bauchy, A. Kachmar, and M. Micoulaut, J. Chem. Phys. 141, 194506 (2014)

[55] W. A. Kamitakahara, R. L. Cappelletti, P. Boolchand, B. Halfpap, F. Gompf, D. A. Neumann, and H. Mutka, Phys. Rev. B 44, 94 (1991).

[56] J. J. Kim, K. Kobayashi, E. Ikenaga, M. Kobata, S. Ueda, T. Matsunaga, K. Kifune, R. Kojima, and N. Yamada, Phys. Rev. B 76, 115124 (2007).

[57] M. Bauchy, M. Micoulaut, M. Boero, and C. Massobrio, Phys. Rev. Lett. 110, 165501 (2013). 